\title{
Introduction to the ISO Specification Language LOTOS
}

\section{Tommaso BOLOGNESI}

CNUCE-C.N.R., Via S. Maria 36, 56100 Pisa, Italy (earn: bolog (aicnucevm)

\section{Ed BRINKSMA}

University of Twente, P.O. Box 217, 9700 AE Enschede, The Netherlands (uucp: mcvax!utinul!infed; earn: hiddink@hentht5)

LOTOS is a specification language that has been specifically developed for the formal description of the OSI (Open Systems Interconnection) architecture, although it is applicable to distributed, concurrent systems in general. In LOTOS a system is seen as a set of processes which interact and exchange data with each other and with their environment. LOTOS is expected to become an ISO international standard by 1988 .

Keywords: Concurrent Languages, Formal Description Techniques, Open Systems Interconnection, Protocol Specification, Specification Languages.

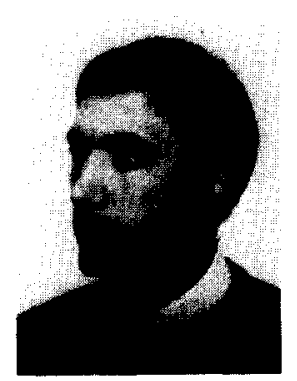

Tommaso Bolognesi (Laurea in Physics, University of Pavia, 1976 M.Sc. in Computer Science, Univ. of Illinois, 1982) joined CNUCE-C.N.R. in 1982, where he is working on specification languages and verification techniques for concurrent systems. In 1984-5 he has been visiting scientist at the IBM Research Laboratory, Zurich, where he has studied verification techniques for time-dependent communication protocols. He has participated to the ISO activities for the definition of LOTOS since 1984, and has collaborated with the University of Twente on the L.OTOS part of the ESPRIT project SEDOS, from 1984 to 1987.

North-Holland

Computer Networks and ISDN Systems 14 (1987) 25-59

\section{Introduction}

LOTOS (Language of Temporal Ordering Specification) is one of the two Formal Description Techniques [26,27] developed within ISO (International Organization for Standardization) for the formal specification of open distributed systems, and in particular for those related to the Open Systems Interconnection (OSI) computer network architecture $[24,39]$. It was developed by FDT experts from ISO/TC97/SC21/WG1 ad hoc group on FDT/Subgroup C during the years 1981-86. The basic idea that LOTOS developed from was that systems can be specified by defining the temporal relation among the interactions that constitute the externally observable behaviour of a system. Contrary to what the name seems to suggest, this description technique is not related to temporal logic, but is based on process algebraic methods. Such methods were first introduced by Milner's work on CCS [33], soon to be followed by many closely related theories that are often collectively referred to as process algebras, e.g. $[2,5,22,32,35]$. More specifically the component of LOTOS that deals with the description of process behaviours and interactions has borrowed many ideas from $[22,33]$.

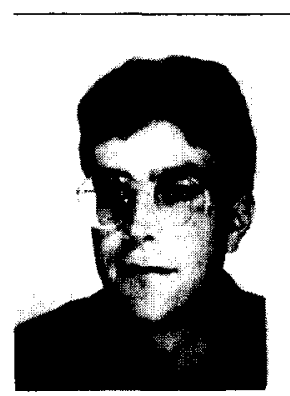

Ed Brinksma studied mathematics (mathematical logic) at the State University of Groningen, The Netherlands, after which he joined the Computer Science group of that university. In 1982 he became an assistent professor at the Computer Science department of the University of Twente, The Netherlands, where he joined the IPS group of computer network research. His main research interest is in the formal specification, verification and testing theory of complex distributed systems. Since 1983 Ed Brinksma has been the chairman of the ISO group responsible for the definition of LOTOS (ISO/TC97/SC21/WG1/FDT/C). He is the editor of the current Draft International Standard (DIS 8807) for LOTOS. In the ESPRIT/SEDOS project (1984-1987) he was the manager of the subtask devoted to the development of verification methods and tools for LOTOS. 
LOTOS also includes a second component, which deals with the description of data structures and value expressions. This part of LOTOS is based on the formal theory of abstract data types, and in particular the approach of equational specification of data types, with an initial algebra semantics (see, e.g. [16]). Most concepts in this component were inspired by the abstract data type technique ACT ONE [16], although there are a number of differences.

LOTOS is an FDT generally applicable to distributed, concurrent, information processing systems. However, it has been developed particularly for OSI. The main objectives for such a technique is that it should allow the production of OSI standards specifications that are:

- unambiguous, precise, complete and implementation independent descriptions of the standards;

- readable documents for OSI users, implementers and conformance testers;

- a formally well-defined basis for the verification and validation of the standards, and for the conformance testing of their implementations.

It is clear that these objectives are particularly important of a distributed, standard architecture, such as OSI. Machines must communicate and cooperate with each other, and informal, ambiguous specifications of the related software could easily lead to incompatible implementations. Furthermore, the possibility to carry out rigorous analysis of a protocol at the design level, that is, in an early stage of the development cycle, is crucial to avoid the proliferation of errors in the expectedly large number of its implementations.

The consideration of the requirements above has led to a number of design criteria for the language itself. The general criteria that have determined the present definition of LOTOS are:

- Expressive power: an FDT should be capable of expressing the wide range of properties that are relevant for the description of OSI services, protocols and interfaces.

- Formal definition: syntax and semantics of an FDT should have a complete and formal definition. In particular, the formal model on which the semantics of the language is based must support the development of an analytical theory for verification, validation and conformance testing.

- Abstraction: the language constructs should represent the relevant architectural concepts at a sufficiently high level of abstraction, where implementation oriented details are not expressed. This avoids the specification of undesirable constraints on implementers, and favours of a precise representation of the requirements.

- Structure: and FDT should offer means for structuring a specification in a meaningful and intuitively pleasing way. Good structuring implies readability, ease of maintenance, and may simplify the analysis. If desirable, structure may also be used to reflect the logical or even physical organization of an implementation.

LOTOS is expected to become ISO international standard by 1988 .

The layout of the paper is as follows. Section 1 is meant to introduce informally the basic elements of the underlying model of LOTOS, namely processes, their interactions and their composition, in order to provide an intuitive support for their formal treatment in the rest of the paper. Basic LOTOS is introduced in Section 2. This is the subset of LOTOS where processes interact with each other by pure synchronizations, without exchanging values. In basic LOTOS we can appreciate the expressiveness of all the LOTOS process constructors (operators) without being distracted by interprocess value communication. Section 3 deals with equivalences, which are important for comparing specifications, and for giving a complete, formal semantics to the language. Value communication is not necessary to treat equivalences, and for this reason we introduce them right after Section 2 on basic LOTOS. The reader only interested in the "surface" of the language may safely skip this section.

The way data values are defined and expressed is the subject of Section 4 on data types. In Section 5 value expressions are integrated into the language: processes may exchange these values, or be parametrized by them, and we have full LOTOS. A small but complete LOTOS specification is provided in Section 6, as an example of the so called "constraint-oriented" specification style. Section 7 contains some concluding remarks and a number of pointers to the literature on LOTOS applications and tools. Already some tutorials on LOTOS have been published (e.g. [7]). These however still refer to previous versions of the language and/or are less complete in their presentation. 


\section{Process}

In LOTOS a distributed, concurrent system is seen as a process, possibly consisting of several sub-processes. A sub-process is a process in itself, so that in general a LOTOS specification describes a system via a hierarchy of process definitions. A process is an entity able to perform internal, unobservable actions, and to interact with other processes, which form its environment. Complex interactions between processes are built up out of elementary units of synchronization which we call events, or (atomic) interactions, or simply actions.

Events imply process synchronization, because the processes that interact in an event (they may be two or more) participate in its execution at the same moment in time. Such synchronizations may involve the exchange of data. Events are atomic in the sense that they occur instantaneously, without consuming time. An event is thought of as occurring at an interaction point, or gate, and in the case of synchronization without data exchange, the event name and the gate name coincide.

The environment of a process $P$, in a system $S$, is formed by the set of processes of $S$ with which $P$ interacts, plus an unspecified, possibly human, observer process, which is assumed to be always ready to observe anything observable the system may do. And, to be consistent with the model, observation is nothing but interaction. Hence, when we say that process $\mathrm{P}$ performs an observable action we refer to the interaction between $\mathrm{P}$ and, at least, the observer. (Note that although we use the words action and interaction as synonyms, we may prefer one or the other depending on the context: we talk about the action performed by one process and the interaction involving $n$ processes. The reason for blurring this distinction is simply that $n$ processes together can be seen as one process.)

The most abstract representation of process $P$, able to interact with its environment via gates, say, $a$ through $g$, is the black box in Fig. 1 .

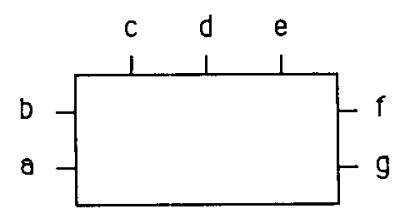

Fig. 1. Process $P$ with gates $a$ through $g$, as a black box.
The process definition of $\mathrm{P}$ will then specify its behaviour, by defining the sequences of observable actions that may occur (be observed) at the seven gates of the process. We will soon represent such behaviour as a tree of actions.

Black boxes are the traditional intuitive representation for process. Vending machines are also used, sometimes, to give a more concrete model of processes and interactions [22]. As another variant of the black box concept we introduce here a music instrument, to be called proto-pianola, where interaction with the environment is achieved via a keyboard. Speaking about these devices turns out to be essentially the same thing as speaking about LOTOS processes.

The proto-pianola fills a gap between the piano and pianola. A piano is a completely passive instrument, since it plays only when its keys are pressed; conversely, a pianola is active, in that it includes a predefined score (on punched paper rolls) and is able of automatically performing it. The proto-pianola is active and passive at the same time: it needs external interaction at the keyboard, for playing, and yet it possesses an internal score, and is able, from time to time, to perform autonomous choices. Figure $2 \mathrm{a}$ represents a 7-key proto-pianola. We can immediately think of it as a LOTOS process, called, say, PP1, and write:

$\mathrm{PP} 1[a, b, c, d, e, f, g]$

to indicate that the 7 keys are the gates through which the process interacts with its environment. In this case the environment, or the observer, is a player. The LOTOS view that processes cannot engage in more than one interaction at a time is reflected by the assumption of monophony for our musical instrument: it cannot produce more than one sound at a time.

The pressing of a key is an interaction between the proto-pianola, which is ready to have that key pressed, and the performer/observer, who is ready to press the key. Both parties participate to, or experience that event, and in fact the "genuine" LOTOS point of view insists on this symmetry, without distinguishing between active and passive roles. Observable actions (thus interactions) are simply identified by the gate where they occur (later they will be given more structure, to allow value communication between processes). We will sometimes express the fact that a process is ready 


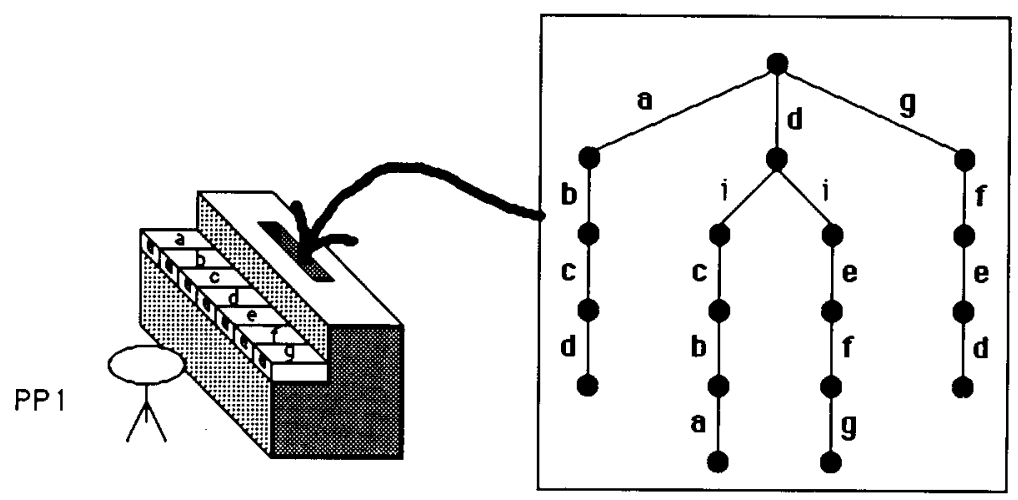

(a)

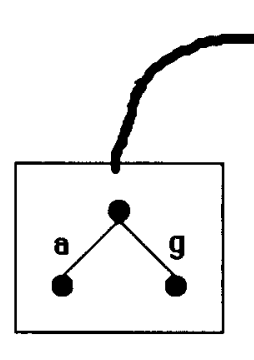

(c) (b)

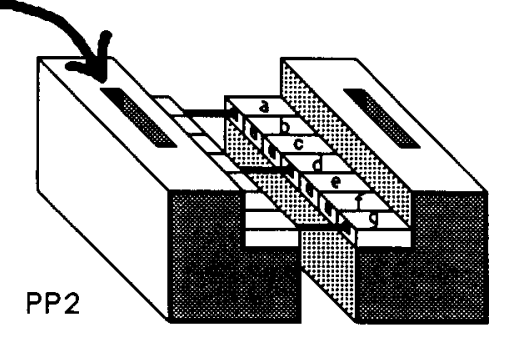

(d)

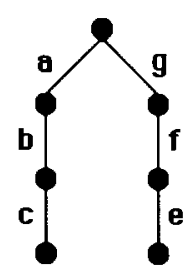

(e)

Fig. 2. Processes as proto-pianolas.

for an interaction at gate $a$ by saying that it offers observable action $a$ to its environment.

A (one-finger) performer sitting at the keyboard of PP1 would not always succeed in pressing a chosen key, since some keys sometimes are locked, and the success in pressing a key depends on the tune played up to that point. The behaviour of the instrument (or, equivalently, its nondeterministic score) is depicted as a tree in Fig. 2b. In this specific case the one-finger performer will be able to play a four note scale, but his only freedom is exercised in the choice of the initial key, which can be $a, d$ or $g$. The other four keys are initially blocked. After the choice of $a$ or $g$, the performer will succeed only in completing his scale, moving, respectively, upwards or downwards. If the initial choice had been for the central key $d$, both directions would have had a chance to be successfully executed, but this choice is not up to the performer. An i-labelled arc in the tree indicates an internal, unobservable action autono- mously performed by the machine, which the performer cannot observe nor hear; the pair of ilabelled branches indicates that the choice of which key becomes unblocked after $d$ is pressed is made by the machine.

The reason for the little holes observed in the front of the proto-pianola keys is revealed by Fig. 1d, where two pianolas, PP1 and PP2, are coupled front to front. Their keyboards appear as mirror images. Metal bars have been inserted into the holes to couple some of the keys. Again a performer is supposed to play the trial and error game on the resulting "keyboard". His success in pressing an independent (uncoupled) key of pianola PP1 (PP2) depends only the "score" of PP1 (PP2), but for a double-key (a pair of coupled keys) to be unlocked both scores must agree, at that point, on the executability of that note. This is exactly the idea of parallel composition of processes in LOTOS. The appropriate behaviour expression would be: 
$\mathrm{PP} 1[a, b, c, d, e, f, g]$

$|[a, d, g]|$

$\mathrm{PP} 2[a, b, c, d, e, f, g]$

where ' $|[a, d, g]|$ ' is a parallel composition operator: the two processes are coupled via the synchronization gates $a, d$ and $g$, thus they may (in fact, must) synchronize only at these gates. The pair of coupled proto-pianolas is essentially a new instrument, and its behaviour is again representable by a tree. Suppose that the behaviour of PP2 be the one in Figure 1c. Then the behaviour of the new, double instrument, with the indicated key couplings, would be as in Fig. 1e: only two threenote tone rows are allowed, starting from either extreme of the keyboard.

Observing and composing LOTOS processes is basically like playing and coupling proto-pianolas. However, since LOTOS has been mainly designed for specifying communication protocols for computer networks, it also includes features which the inventors of the proto-pianola failed to anticipate. One of these is hiding. This feature is better introduced by going back to the non-musical, more abstract world of black-boxes, where a process is represented as in Figure 1.

Consider Figure 3. The intended interpretation of the depicted system is as follows. Process Max3 is defined by composing in parallel two instances of process Max2. Each one of the component processes may interact with its own environment, which consists of the other instance of Max2 and the outer environment, via three gates; but the only synchronization gate between the two processes is mid. Notice that this gate is included in the outer box which represents process Max3. Since we insist that this box is really black, the gate is not visible from the outer environment: it has been hidden. The two process instances are thus allowed to independently interact with the

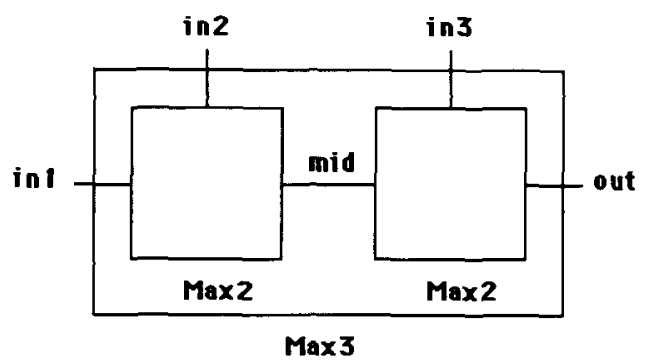

Fig. 3. Spatial representation of process Max3. environment at all gates except mid. At this gate they are required to synchronize with each other, without the environment observing (taking part in) these interactions: due to the hiding, these interactions have become internal actions of the system. The informal description above is made formal below.

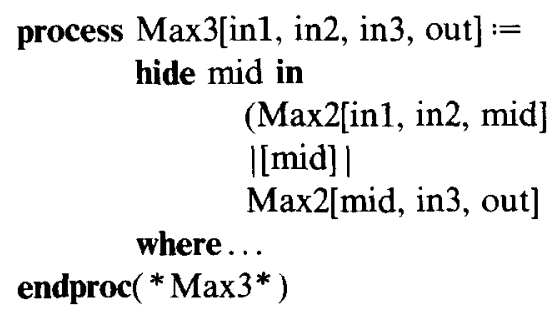

The fact that the system may interact with its environment via actions (at gates) inl, in2, in 3, $o u t$, is explicitly indicated in the first line of the specification. Since gate mid is hidden, by the hide operator, it does not appear in that list.

The partial specification above is complemented in the next section.

\section{Basic LOTOS}

Basic LOTOS is a simplified version of the language employing a finite alphabet of observable actions. This is so because observable actions in basic LOTOS are identified only by the name of the gate where they are offered, and LOTOS processes can only have a finite number of gates. Three examples of observable actions that we have already met in the previous section are:

$\mathrm{g}$

in2

out

The structure of actions will be enriched in full LOTOS by allowing the association of data values to gate names, and thus the expression of a possibly infinite alphabet of observable actions.

Basic LOTOS only describes process synchronization, while full LOTOS also describes interprocess value communication. In spite of this remarkable difference, we will initially concentrate on basic LOTOS for three reasons. First, within this proper subset of the language we can appreciate the expressiveness of all the LOTOS process constructors (operators) without being distracted by interprocess communication; second, for basic 
LOTOS we can give an elegant and, most importantly, formal presentation of the semantics, without boring the reader with cumbersome notation; third, behavioural equivalences are more conveniently introduced at this level. Full LOTOS will be introduced only in Section 5 .

\subsection{Process Definitions and Behaviour Expressions}

The typical structure of basic LOTOS process definition is given in Fig. 4, which completes the definition of process Max3 started in Section 1. As a convention we will use italics for syntactic categories, that is, nonterminal symbols (e.g. behaviour expression), and boldface for reserved LOTOS keywords (e.g. process).

An essential component of a process definition is its behaviour expression. A behaviour expression is built by applying an operator (e.g., '[ ]') to other behaviour expressions. A behaviour expression may also include instantiations of other processes (e.g. Max2), whose definitions are provided in the where clause following the expression. Given behaviour expression B, we will allow calling B also "a process", for convenience, even when no process name is explicitly associated with the behaviour expressed by B.

The complete list of basic-LOTOS behaviour expressions is given in Table 1, which includes all
Table 1

Syntax of behaviour expressions in basic LOTOS

\begin{tabular}{ll}
\hline Name & Syntax \\
\hline $\begin{array}{l}\text { inaction } \\
\text { action prefix }\end{array}$ & stop \\
$\quad$-unobservable (internal) & $\mathrm{i} ; \mathrm{B}$ \\
$\quad$ observable & $\mathrm{g} ; \mathrm{B}$ \\
choice & $\mathrm{B} 1[] \mathrm{B} 2$ \\
parallel composition & \\
$\quad$-general case & $\mathrm{B} 1 \|\left[\mathrm{g}_{1}, \ldots, \mathrm{g}_{\mathrm{n}}\right] \mid \mathrm{B} 2$ \\
$\quad$-pure interleaving & $\mathrm{B} 1 \| \mathrm{B} 2$ \\
$\quad$ full synchronization & $\mathrm{B} 1 \| \mathrm{B} 2$ \\
hiding & hide $\mathrm{g}_{1}, \ldots, \mathrm{g}_{\mathrm{n}}$ in $\mathrm{B}$ \\
process instantiation & $\mathrm{p}\left[\mathrm{g}_{1}, \ldots, \mathrm{g}_{\mathrm{n}}\right]$ \\
successful termination & exit \\
sequential composition (enabling) & $\mathrm{B} 1 \gg \mathrm{B} 2$ \\
disabling & $\mathrm{B} 1[>\mathrm{B} 2$ \\
\hline
\end{tabular}

basic LOTOS operators. Symbols 'B', 'B1', 'B2' in the table stand for any behaviour expression. Any behaviour expression must match one of the formats listed in column Syntax. We have taken the metalinguistic liberty of representing some lists with dots. By inspecting Table 1 we may observe that basic LOTOS includes nullary operators (e.g. inaction), unary operators (e.g. action prefix) and binary operators (e.g. parallel composition), that is, operators applicable to, respectively, none, one and two behaviour expressions.

Operator precedences are as follows:

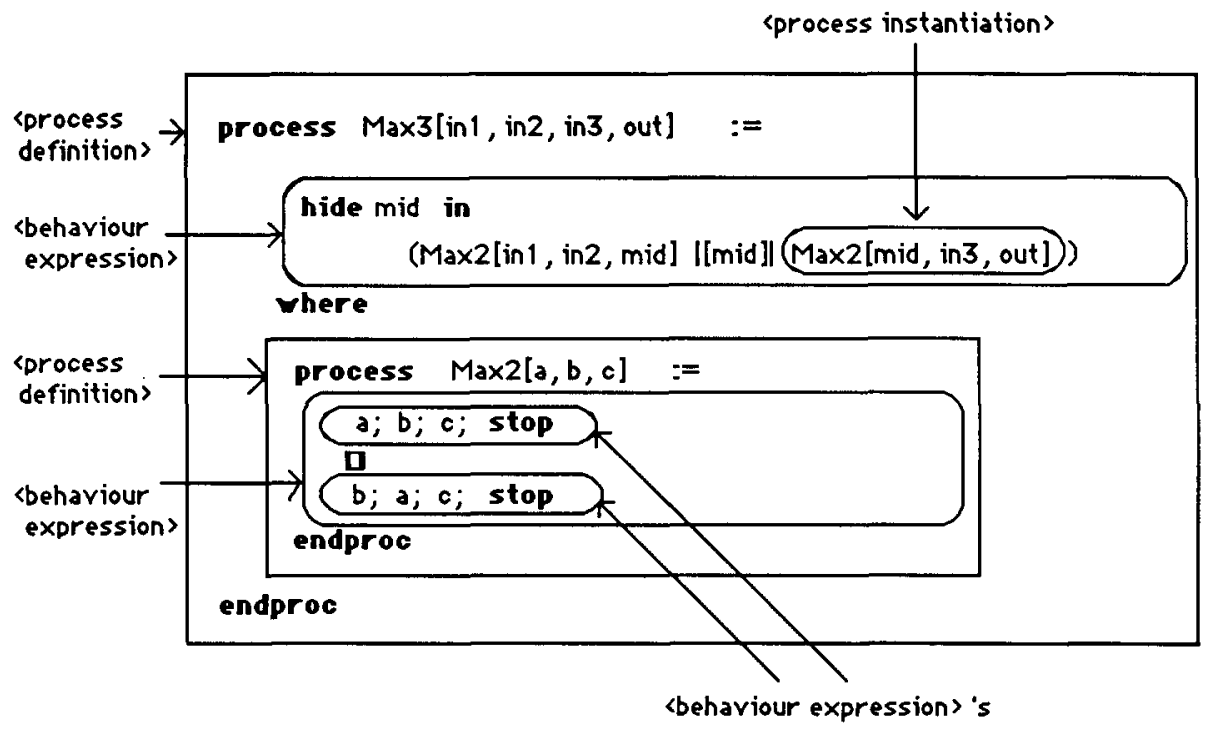

Fig. 4. Definition of process Max3. 
action prefix $>$ choice $>$ parallel composition

$$
>\text { disabling }>\text { enabling }>\text { hiding }
$$

This means that, for example, expression

hide $\mathrm{a}$ in $\mathrm{a} ; \mathrm{P}[\mathrm{Q} \mathrm{Q} \gg \mathrm{R} \| \mathrm{S}[>\mathrm{T}$

is equivalent to expression

hide $\mathrm{a}$ in $(((\mathrm{a} ; \mathrm{P})[] \mathrm{Q})>((\mathrm{R} \| \mathrm{S})[>\mathrm{TT}))$.

\subsection{A Basic Process, Two Basic Operators}

\section{Inaction: stop}

The completely inactive process is represented by stop. It cannot offer anything to the environment, nor it can perform internal actions, and it is as basic in LOTOS as the number zero in arithmetic. Notice that stop can be interpreted as the behaviour expression obtained by applying the nullary operator stop to zero arguments.

Action prefix: i; B

$$
\mathrm{g} ; \mathrm{B}
$$

This is a unary prefix operator which produces a new behaviour expression out of an existing one, by prefixing the latter with an action (gate name) followed by a semicolumn. Examples of action prefix behaviour expressions, taken from process Max3 (Fig. 4) are:

c; stop

b; c; stop

a; b; c; stop

Choice: B1 [] B2

If $\mathrm{B} 1$ and $\mathrm{B} 2$ are two behaviour expressions then B1 [] B2 denotes a process that behaves either like B1 or like B2. The choice offered is resolved in the interaction of the process with its environment. If (another process in) the environment offers an initial observable action of B1, then B2 may be selected. If an action is offered from the environment that is initial to both B1 and B2, then the outcome is not determined. An example of a choice behaviour expression, again taken from Max3, is

$\mathrm{a} ; \mathrm{b} ; \mathrm{c} ; \operatorname{stop}[] \mathrm{b} ; \mathrm{a} ; \mathrm{c} ; \mathbf{s t o p}$

On the basis of the three constructs above, the behaviour of process Max2[a, b, c] defined in Fig. 4 is now clear. As we did for proto-pianolas, we can immediately build the tree of actions associated with this expression. However, it is now time to describe the construction of action trees in a more precise, formal way, which could be systematically applied to any behaviour expression.

\subsection{Operational Semantics: Growing Trees from Expressions}

The operational semantics [38] of LOTOS provides a means to systematically derive the actions that a process (behaviour expression) may perform from the structure of the expression itself. More precisely, given an expression $\mathrm{B}$, what we derive are labelled transitions, that is triples of type:

$\mathrm{B}-\mathrm{x} \rightarrow \mathrm{B}^{\prime}$

where $\mathrm{x}$ is an action and $\mathrm{B}^{\prime}$ is another behaviour expression: $\mathrm{B}$ may perform action $\mathrm{x}$ and transform into $B^{\prime}$. In defining the semantics we will let:

$G$ denote the set of user-definable gates;

$\mathrm{g}, \mathrm{g}_{1}, \ldots, \mathrm{g}_{\mathrm{n}}$ range over $\mathrm{G}$;

i denote the unobservable action;

Act denote the set $\mathrm{G} \cup\{\mathbf{i}\}$ of user-definable actions;

$\mu \quad$ range over Act.

Furthermore, we will need to handle a special action (gate) ' $\delta$ ', which is not user-definable, and whose occurrence indicates the successful termination of a process and the enabling of a subsequent process. We will thus let:

$\delta \quad$ be the successful termination action

$\mathrm{G}^{+} \quad$ be the set $\mathrm{G} \cup\{\delta\}$ of observable actions

$\mathrm{g}^{+} \quad$ range over $\mathrm{G}^{+}$

Act $^{+}$be the set Act $\cup\{\delta\}$ of actions

$\mu^{+} \quad$ range over Act $^{+}$.

If $B E$ is the set of behaviour expressions, then we may say, more formally, that the axioms and inference rules of the operational semantics allow the definition of the labelled transition relation ' $\rightarrow$ ', which is a subset of $B E \times \mathrm{Act}^{+} \times B E$ (' $\times$ ' is the cartesian product of sets). By applying axioms and rules to a given expression we build the transition tree, also called synchronization tree. (An introduction to these topics, and to the way axioms and inference rules are used, can be found by the interested reader also in [33], which gives the operational semantics of CCS). In a transition tree nodes are labelled by behaviour expressions (the starting expression being the label of the root), and arcs are labelled by actions. An action tree is a transition tree where node labels have been deleted. 
Despite the tutorial nature of this paper, we found appropriate to present the semantics in a formal way, because, in this case, the formalism directly and naturally reflects our intuitive understanding of the meaning of expressions; and the little cost of explaining how to read axioms and inference rules is more than compensated by the advantages in terms of clarity and conciseness. In fact, since the beginning of the ISO/FDT activities, the definition of a formal semantics has been considered as a major requirement in defining Formal Description Techniques.

\section{Semantics of Inaction, Action Prefix and Choice}

No axiom or inference rule is associated with behaviour expression stop, and it is thus impossible to derive any transition from it. Hence we understand stop as a predefined LOTOS process which is unable to perform any action or to interact with any other process.

The semantics of the action prefix behaviour expression is captured by a single axiom:

$$
\mu ; \mathrm{B}-\mu \rightarrow \mathrm{B}
$$

where $\mathbf{B}$ is any behaviour expression and $\mu$ is either the unobservable action $\mathbf{i}$ or some observable action $g$. This axiom states the true fact, subject to no condition, that process ' $\mu$; $\mathrm{B}$ ' is capable of performing action $\mu$ and transform into process B. Notice that we use $\mu$ and not $\mu^{+}$. This is because the user of the language is not allowed to express the successful termination action ' $\delta$ ' directly, but only indirectly, by the 'exit' construct (to be discussed later).

B1 [] B2 is a choice behaviour expression which behaves either like B1 or like B2. Its behaviour is captured by the two inference rules:

$$
\begin{aligned}
& \mathrm{B} 1-\mu^{+} \rightarrow \mathrm{B} 1^{\prime} \text { implies } \mathrm{B} 1[] \mathrm{B} 2-\mu^{+} \rightarrow \mathrm{B} 1^{\prime} \\
& \mathrm{B} 2-\mu^{+} \rightarrow \mathrm{B} 2^{\prime} \text { implies } \mathrm{B} 1[] \mathrm{B} 2-\mu^{+} \rightarrow \mathrm{B} 2^{\prime}
\end{aligned}
$$

These rules are used to derive the actions of B1 [] B2 from those of B1 or B2. More precisely, the action capability (set of possible actions) of a choice expression is the union of the action capabilities of its components; however, once an action is chosen from one component, the other component disappears from the resulting expression.

If we apply the axiom for action prefix, for example, to expression ' $a$; $b$; $c$; stop' we obtain the transition:

$\mathrm{a} ; \mathrm{b}$; c; stop - a $\rightarrow \mathrm{b}$; c; stop

We may now use this result in applying the inference rule for choice:

$\mathrm{a} ; \mathrm{b} ; \mathrm{c}$; stop - a $\rightarrow \mathrm{b}$; ; stop

implies

a; b; c; stop [ ] b; a; c; stop - a $\rightarrow$ b; c; stop

We have thus derived a transition for a choice expression, based on the operational semantics of the language. By exhaustively applying the axiom and the rules in all possible ways, the reader may easily find the seven-node tree associated to the choice expression above.

As a final example of inaction, action prefix and choice we give the basic LOTOS description of the process illustrated in Fig. 5. This process describes the externally observable behaviour of a full-duplex channel between two points, which can be used only once for each direction. The description is abstract, in the sense that it only accounts for the ordering of inputs and outputs, and not for the data actually transmitted.

process duplex-buffer [in-a, in-b, out-a, out-b] := in-a; (in-b; (out-a; out-b; stop []out-b; out-a; stop)

[ ]out-a; in-b; out-b; stop)

[] in-b; (in-a; (out-a; out-b; stop [] ]out-b; out-a; stop)

[ ]out-b; in-a; out-a; stop)

\section{endproc}

As it appears from the example above, describing a behaviour using only inaction, action prefix and choice forces for the specifier to explicitly describe all different orderings in which independent actions may take place. This is of course a rather clumsy solution, and we will show below how parallel composition solves this problem in a more concise and structured way.

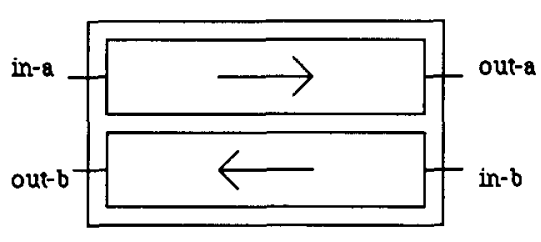

Fig. 5. A simple, full-duplex buffer. 


\subsection{Parallelism}

General case: $\mathrm{B} 1\left|\left[\mathrm{~g}_{1}, \ldots, \mathrm{g}_{\mathrm{n}}\right]\right| \mathrm{B} 2$

Let $S=\left[g_{1}, \ldots, g_{n}\right]$ be a set of user-defined gates, called synchronization gates. Given a parallel behaviour expression ' $\mathrm{B} 1|\mathbf{S}| \mathbf{B} 2$ ', the transitions is can perform depend on the transition capabilities of $\mathrm{B} 1$ and $\mathrm{B} 2$, and on $\mathrm{S}$, as expressed by the following inference rules:

$\mathrm{B} 1-\mu \rightarrow \mathrm{B}^{\prime}$ and $\mu \notin \mathrm{S}$
implies
$\mathrm{B} 1|\mathrm{~S}| \mathrm{B} 2-\mu \rightarrow \mathrm{B} 1^{\prime}|\mathrm{S}| \mathrm{B} 2$
$\mathrm{~B} 2-\mu \rightarrow \mathrm{B} 2^{\prime}$ and $\mu \notin \mathrm{S}$
implies
$\mathrm{B} 1|\mathrm{~S}| \mathrm{B} 2-\mu \rightarrow \mathrm{B} 1|\mathrm{~S}| \mathrm{B} 2^{\prime}$
$\mathrm{B} 1-\mathrm{g}^{+} \rightarrow \mathrm{B} 1^{\prime}$ and $\mathrm{B} 2-\mathrm{g}^{+} \rightarrow \mathrm{B} 2^{\prime}$
and $\mathrm{g}^{+} \in \mathrm{S} \cup\{\delta\}$
implies
$\mathrm{B} 1|\mathrm{~S}| \mathrm{B} 2-\mathrm{g}^{+} \rightarrow \mathrm{B} 1^{\prime}|\mathrm{S}| \mathrm{B} 2^{\prime}$

The rules essentially say that a parallel composition expression is able to perform any action that either component expression is ready to perform at a gate not in $S$ (excluding successful termination ' $\delta$ '), or any action that both components are ready to perform at a gate in $S$, or at gate $\delta$. This implies that when process $\mathrm{B} 1$ is ready to execute some action at one of the synchronization gates, it is forced, in the absence of alternative actions, to wait until its "partner" process B2 offers the same action.

As an example, consider the parallel behaviour expression

$$
\begin{aligned}
& \text { 'Max2[in1, in2, mid }] \\
& |[\mathrm{mid}]| \\
& \operatorname{Max} 2[\mathrm{mid}, \text { in } 3, \text { out }]
\end{aligned}
$$

used to define the behaviour of process Max3 in Fig. 4. The action trees associated with the two instances of process Max2 are given in Fig. 6. They are easily obtained by first building the transition tree of process Max2, also defined in Fig. 4, and then by properly replacing its $a, b, c$ labels with the actual gate names used in the two process instantiations (we are giving an informal preview of the semantics of process instantiation).

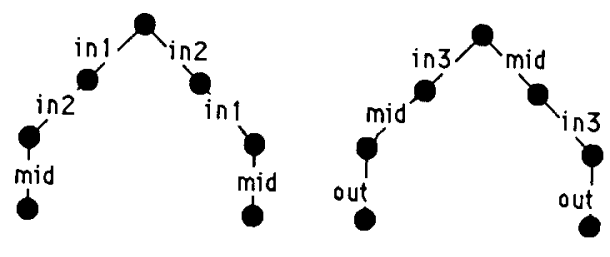

Fig. 6. Two action trees.

By repeatedly applying the inference rules for parallel composition, the reader may check that the action tree for the parallel expression is above is as depicted in Fig. 7.

Notice that action mid is not hidden, as it was in the definition of process Max3. Thus, it is available for further synchronizations with the environment, exactly as is the case for actions inl, in2, in 3 and out. This feature of multi-process or multi-way synchronization is important for both technical and methodological reasons. The technical reasons have to do with specific applications. In some applications the structure of interprocess communication is reflected best by specifying a multi-way synchronization between processes. This is the case with, for example, message broadcasting.

The methodological reasons for introducing multi-way synchronization are related to the fact that where many processes synchronize on a single action, each of these processes may add constraints with respect to the occurrence of that action. In other words, complex temporal ordering relations among actions may be decomposed as the conjunction of several simpler constraints, each of which may be captured by a simple process definition. The complex constraint is then expressed by the parallel composition of all these simpler processes. This method is referred to as constraint-oriented specification. We illustrate this with a small example.

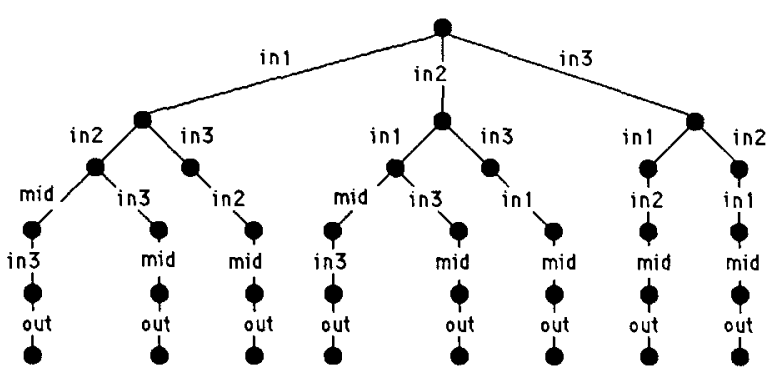

Fig. 7. A parallel composition of the two action trees of Fig. 6. 
Consider (again!) the behaviour expression defining process $\operatorname{Max} 2[a, b, c]$ :

a; b; c; stop [] b; a; ; stop

This process offers actions $a$ and $b$, in either order, followed by action c. We may equivalently say that the only temporal constraints involved are

"a precedes c" and "b precedes c",

where the ' $c$ ' in the two constraints has to be regarded as a unique action. The conjunction of these two constraints is precisely expressed by the parallel composition operator as follows:

a; c; stop $|[c]| b ; c$; stop

In fact, the action trees for the two expressions above turn out to be identical.

This approach of 'logical modularity' allows for an incremental combination of constraints. A further constraint such as " $x$ precedes $c$ " can be added later, with no need to affect the expression built so far:

(a; c; stop $|[\mathrm{c}]| \mathrm{b} ; \mathrm{c} ;$ stop) $|[\mathrm{c}]| \mathrm{x}$; c stop

There exist two special cases of the parallel operator, for which convenient shorthands are defined. They are called pure interleaving and full synchronization.

\section{Pure interleaving $\quad B 1|| \mid B 2$}

When the set of synchronization gates, $\mathrm{S}$, is empty, the parallel operator ' $|S|$ ' is written ' $\| \mid$ '. By inspecting the inference rules for parallel composition, it is clear that in this case the third rule can never be applied, except in the case of successful termination.

The two rules left account for the actions performed by the two component processes independently of each other. Given expression B1 || B2, if both $\mathrm{B} 1$ and $\mathrm{B} 2$ are ready for some action (say actions $b 1$ and $b 2$ respectively), then both action orderings ( $b 1$ before $b 2, b 2$ before $b 1$ ) are possible. Notice that $b 1$ and $b 2$ may even be the same. Since B1 |||B2 transforms, after an action, into an expression still involving the ' $\| \mid$ ' operator, we conclude that this case of parallel composition expresses nothing but any interleaving of the actions of B1 with the actions of B2.

We have now a means for expressing the simple-duplex-buffer specified at the end of Section 2.3. As suggested by Fig. 5, such a process is best represented by a parallel composition of two inde- pendent processes (buffers). A more concise and better structured specification is:

process duplex-buffer [in-a, in-b, out-a, out-b] := simplex-buffer [in-a, out-a]

where

III simplex-buffer [in-b, out-b]

process simplex-buffer [in, out] := in; out; stop

endproc

endproc

Full synchronization $\quad B 1 \| B 2$

When the set of synchronization gates, $\mathrm{S}$, is the set $\mathrm{G}$ of all gates, then the parallel operator ' $|\mathrm{S}|$ ' is written ' $\|$ '. Only the third inference rule for the parallel operator is applicable for actions in $\mathrm{G}^{+}$, and the two composed processes are forced to proceed in complete synchrony except for possible internal actions.

A typical example of use of this parallel operator is when the capabilities of a process are determined by two or more of its subprocesses.

process produce $[\mathrm{a}, \mathrm{b}, \mathrm{c}, \mathrm{d}]:=$ item-available $[a, b, c, d]$

\| item-acceptable [a, b, c, d] where

process item-available $[a, b, c, d]:=$ a; (b; item-available $[\mathrm{a}, \mathrm{b}, \mathrm{c}, \mathrm{d}]$ [] c; item-available $[a, b, c, d]$

endproc )

process item-acceptable $[\mathrm{a}, \mathrm{b}, \mathrm{c}, \mathrm{d}]:=$ a; (b; item-acceptable

$[\mathrm{a}, \mathrm{b}, \mathrm{c}, \mathrm{d}]$

[] d; item-acceptable

$[a, b, c, d]$

endproc )

endproc

In this simple example we can check that "produce[a, b, c, d]' may only perform the sequence of actions ' $a, b, a, b, \ldots$ '. (For the use of recursion, see Section 2.6.)

\subsection{Hiding}

Hiding allows one to transform some observable actions of a process into unobservable ones. These action are thus made unavailable for syn- 
chronization with other processes. The inference rules for the hiding operator are:

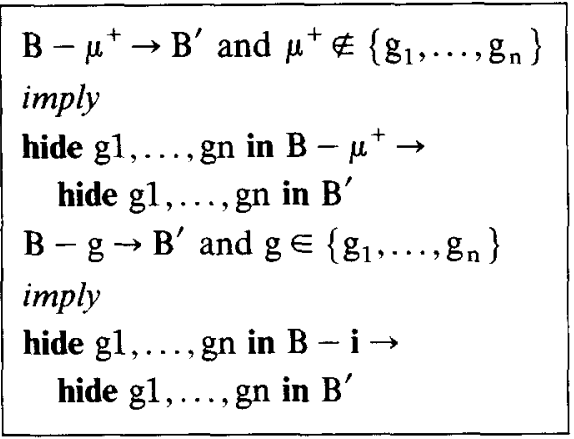

Any action occurring at a gate in the set of hidden gates is transformed into an $\mathrm{i}$-action (second rule). Any other action, including ' $\mathbf{i}$ ' and successful termination, is unaffected by the operator (first rule). We may say that hiding introduces unobservable actions in a specification implicitly, while by action prefix they can be introduced explicitly.

As an example, consider the hiding behaviour expression

$$
\begin{aligned}
& \text { hide mid in } \\
& \operatorname{Max} 2[\text { in } 1, \text { in } 2, \text { mid }] \\
& \mid[\text { mid }] \mid \\
& \operatorname{Max} 2[\mathrm{mid}, \text { in } 3, \text { out }]
\end{aligned}
$$

used to define the behaviour of process Max3 in Fig. 4. The hiding operator makes the synchronization between the two Max 2 processes invisible, and excludes interference from their environment. We do this since we know that no other process will be added later to impose further temporal constraints to the occurrence of the mid action, which is to be considered as a "private" interaction between the two instances of Max2.

The action tree for the expression above is directly obtained from the tree of expression 'Max2[in1, in2, mid] |[mid] |Max2[mid, in3, out]', given in Fig. 7 , by replacing the mid labels with i-labels. We will use this tree later (it can be found in Fig. 12b).

\subsection{Process Instantiation and Recursion}

A process instantiation ' $\mathrm{P}[\mathrm{g} 1, \ldots, \mathrm{gn}]$ ' is formed by a process identifier ' $\mathrm{P}$ ' with an associated list $\left[\mathrm{g}_{1}, \ldots, \mathrm{g}_{\mathbf{n}}\right]$ of actual gates. Such a process instantiation occurs in the behaviour expression defining some other process, or process $\mathrm{P}$ itself. The instantiation of a LOTOS process resembles the invocation of a procedure in a programming language such as Pascal. Of course a process instantiation refers to a process definition which must exist somewhere in the specification, and whose behaviour is defined in terms of a list $\left[\mathrm{g}_{1}^{\prime}, \ldots, \mathrm{g}_{\mathrm{n}}^{\prime}\right]$ of formal gates. Example: in Fig. 4, 'Max2[mid, in 3 , out]' is a process instantiation, where '[mid, in 3 , out]' is a list of actual gates, while ' $[a, b, c]$ ' is the matching list of formal gates.

Although the interpretation of process instantiation is simple, in order to formally define how formal gates are replaced by actual gates we need to introduce an auxiliary operator, called relabelling, which is only used for talking about LOTOS, and not for specifying processes in LOTOS. Relabelling is a unary, postfix operator, which consists of a list of gate-pairs $\left[g_{1} / g_{1}^{\prime}, \ldots, g_{n} / g_{n}^{\prime}\right]$, and is interpreted as gate renaming: gate $g_{i}^{\prime}$ becomes gate $g_{i}, i=1, \ldots, n$. Formally:

$$
\begin{aligned}
& \mathrm{B}-\mathrm{g}^{\prime} \rightarrow \mathrm{B}^{\prime}, \phi=\left[\mathrm{g}_{1} / \mathrm{g}_{1}^{\prime}, \ldots, \mathrm{g}_{\mathrm{n}} / \mathrm{g}_{\mathrm{n}}^{\prime}\right] \\
& \text { and } \mathrm{g} / \mathrm{g}^{\prime} \in \phi \\
& \text { implies } \\
& \mathrm{B} \phi-\mathrm{g} \rightarrow \mathrm{B}^{\prime} \phi \\
& \mathrm{B}-\mu^{+} \rightarrow \mathrm{B}^{\prime} \text { and } \mu^{+} \notin\left\{\mathrm{g}_{1}^{\prime}, \ldots, \mathrm{g}_{\mathrm{n}}^{\prime}\right\} \\
& \text { implies } \\
& \mathrm{B} \phi-\mu^{+} \rightarrow \mathrm{B}^{\prime} \phi
\end{aligned}
$$

Notice that internal action and successful termination are not affected by relabelling. It follows from these rules that the action trees of $B$ and $B \phi$ are the same, except for the renaming of gates which affects some of the arc labels.

The rules for process instantiation are:

$$
\begin{aligned}
& \text { If 'process } P\left[g^{\prime} 1, \ldots, g^{\prime} n\right]:=B_{P} \text { endproc' } \\
& \text { is a process definition then: } \\
& \mathrm{B}_{\mathrm{P}}\left[\mathrm{g}_{1} / \mathrm{g}_{1}^{\prime}, \ldots, \mathrm{g}_{\mathrm{n}} / \mathrm{g}_{\mathrm{n}}^{\prime}\right]-\mu^{+} \rightarrow \mathrm{B}^{\prime} \text { implies } \\
& \quad \mathrm{P}\left[\mathrm{g}_{1}, \ldots, \mathrm{g}_{\mathrm{n}}\right]-\mu^{+} \rightarrow \mathrm{B}^{\prime}
\end{aligned}
$$

where $\left[g_{1} / g_{1}^{\prime}, \ldots, g_{n} / g_{n}^{\prime}\right]$ is the relabelling operator. The behaviour of instantiation ' $\mathrm{P}\left[\mathrm{g}_{1}, \ldots, \mathrm{g}_{\mathrm{n}}\right]$ ' is thus defined as the behaviour of the body $\mathrm{B}_{\mathrm{P}}$ of the associated process definition, with the appropriate gate relabelling. 


\section{Recursion}

Recursion is achieved, in LOTOS, by process instantiation, and is used to express infinite behaviours, namely those which involve action sequences of infinite length. Let us say that "process P invokes process Q" if either an instantiation of $Q$, or the instantiation of another process that invokes $\mathrm{Q}$, occurs in the behaviour expression defining $\mathbf{P}$. We say that process $\mathrm{P}$ is recursive if it invokes itself. As a simple example of recursion (and process instantiation) we refine the definition of the simplex-buffer given at the end of Section 2.4 , by making it reusable:

process reusable-simplex-buffer [in, out] := in; out; reusable-simplex-buffer [in, out] endproc

An infinite sequence in; out; in; out; ... of actions is now possible. Incidentally, an identical behaviour is obtained by the following definition:

process same-simplex-buffer [in, out]:= endproc in; same-simplex-buffer [out, in]

where every new process instantiation inverts the order of gates.

\subsection{Successful Termination and Sequential Com- position}

So far two ways to express sequentiality in specifications are available. We can do it directly, by prefixing an action to a process, or indirectly, by composing in parallel two processes in such a way that the last action of the first process synchronize with the first action of the second one. It seems desirable to have a direct way to express sequential composition of processes too, that is, to have a separate operator for it. This may help in reflecting more clearly the structure of a system into the structure of its specification.

The idea behind the sequential composition operator is that the second process is enabled only if and when the first one terminates successfully.

\section{Successful termination exit}

Exit is a process (a nullary operator, a behaviour expression) whose purpose is solely that of performing the successful termination action $\delta$, after which it transforms into the dead process stop. Its associated axiom is:

exit $-\delta \rightarrow$ stop
Action $\delta$ plays an key role in the sequential composition of processes, as shown below. It cannot be used directly in a specification, but only via the exit construct. Thus any gate accidentally names $\delta$ in a specification is regarded as a "normal" gate, with no termination significance.

Sequential composition $B I \gg B 2$

The informal interpretation of this construct is that if B1 terminates successfully, and not because of a premature deadlock, then the execution of B2 is enabled.

$$
\begin{aligned}
& \mathrm{B} 1-\mu \rightarrow \mathrm{B}^{\prime} \text { implies } \mathrm{B} 1 \gg \mathrm{B} 2-\mu \rightarrow \mathrm{B}^{\prime} \gg \mathrm{B} 2 \\
& \mathrm{~B} 1-\delta \rightarrow \mathrm{B}^{\prime} \text { implies } \mathrm{B} 1 \gg \mathrm{B} 2-\mathrm{i} \rightarrow \mathrm{B} 2
\end{aligned}
$$

The first rule accounts for the behaviour of B1 before its successful termination. The second rule shows that it is action $\delta$, offered by $\mathrm{B} 1$, which enables $\mathrm{B} 2$, and that this passing of control is seen as an internal action $\mathbf{i}$. Sequential composition and hiding are the only operators which introduce unobservable actions implicitly in a specification.

It is important to realize that the successful termination of the parallel composition of two processes is possible if and when both components are ready to successfully terminate, as expressed by the inference rules for the parallel operator. As a negative example consider this expression:

(a, b; exit ||| a; c; stop) $\gg$ second-process [...]

The expression is equivalent to ' $(\mathrm{a} ; \mathrm{b}$; exit $\| \mathrm{a}$; $\mathrm{c}$; stop)', since stop cannot contribute to the successful termination of the parallel subexpression, and no enabling of the second-process takes place.

The enabling operator is conveniently used in conjunction with process instantiation, so that subparts of a system can be first defined as separate processes and then instantiated in the desired sequence. An example is given below.

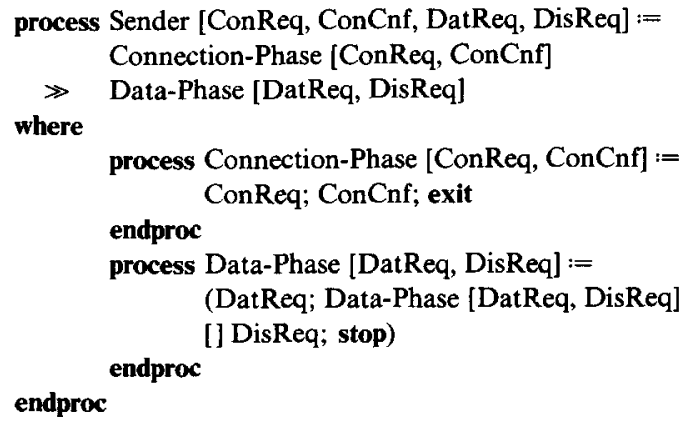




\subsection{Disabling}

In almost any OSI connection oriented protocol or service it is the case that the 'normal' course of action can be disrupted at any point in time by events signalling disconnection or abortion of a connection. This has led to the definition in LOTOS of an 'application generated' operator, namely the disabling operator. Process $\mathrm{B} 1$ may be disabled by process $\mathrm{B} 2$ according to the following rules:

$$
\begin{aligned}
& \mathrm{B} 1-\mu \rightarrow \mathrm{B}^{\prime} \text { implies } \mathrm{B} 1\left[>\mathrm{B} 2-\mu \rightarrow \mathrm{B} 1^{\prime}[>\mathrm{B} 2\right. \\
& \mathrm{B} 1-\delta \rightarrow \mathrm{B}^{\prime} \text { implies } \mathrm{B} 1\left[>\mathrm{B} 2-\delta \rightarrow \mathrm{B} 1^{\prime}\right. \\
& \mathrm{B} 2-\mu^{+} \rightarrow \mathrm{B} 2^{\prime} \text { implies } \mathrm{B} 1\left[>\mathrm{B} 2-\mu^{+} \rightarrow \mathrm{B} 2^{\prime}\right.
\end{aligned}
$$

Process B1 may (third rule) or may not (first and second rules) be interrupted by the first action of process B2. In the first case control is irreversibly transferred from the interrupted $B 1$ to the interrupting $B 2$. In the second case the interruptable $\mathrm{B} 1$ performs an action: if this action is not a successful termination (first rule), B2 survives. If the action is a successful termination (second rule), B2 disappears: the process which B2 was expected to interrupt has terminated, and the disabling process itself is disabled.

As an example, let us first define the two processes:

process Activity $[\mathrm{a}, \mathrm{b}, \mathrm{c}]:=$

$$
\mathrm{a} ; \mathrm{b} ; \mathrm{c} ; \text { Activity }[\mathrm{a}, \mathrm{b}, \mathrm{c}]
$$

\section{endproc}

process Disrupt [discon, reason] := discon; reason; stop

endproc

Then the expression:

Activity[a, b, c] [ $>$ Disrupt[discon, reason]

is equivalent with:

( discon; reason; stop

[] a; ( discon; reason; stop

[] b; ( discon; reason; stop

[] c; (Activity [a, b, c] [> Disrupt [discon, reason]
With disabling, we have completed our presentation of the basic LOTOS operators.

\subsection{Nondeterminism and Internal Actions}

Before giving a final example of a specification in basic LOTOS, we briefly discuss how nondeterminism can be expressed in it. A simple example of nondeterminism is represented by the following expression:

a; b; stop [] a; c; stop

where the result of observing $a$ is not determined. The unobservable action is also a source of nondeterminism, as shown by the expression

i; b; stop [] i; c; stop

(proto-pianola PP1 in Figs. 2(a) and (b) provides a similar example). In fact, from the point of view of an observer who is interested in observing action $b$ (or $c$ ), the two expressions above offer the same uncertainty: in both cases the observation may succeed or fail (but in the first case a preliminary and always successful observation of $a$ is also needed). We discuss now, with an example, the special case of nondeterminism where the alternative is between an observable and an unobservable action.

We want to model a vending machine (although very little remains to be written about these devices after the publication of [22]). After accepting a coin, it will offer some candy. The user can obtain the latter by pulling a drawer.

process Vending_machine [coin, candy1, candy2] $:=$ coin

$$
\text { ;( candy1 }
$$

[]

;Vending_machine [coin, candy1, candy2]

candy 2

;Vending_machine [coin, candy1, candy2] )

endproc

Now suppose the system also contains a little devil that can try at any time to pull a drawer (before the user) and consume the candy.

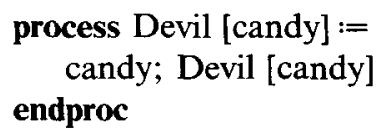

The total system, as observed by the client, is defined by 
process System [coin, candy]:=

hide candy' in

Vending_machine [coin, candy, candy']

[[candy']]

Devil [candy']

\section{endproc}

By applying the axiom and inference rules introduced so far we could start the construction of the action tree for this System. We would then soon realize that the behaviour of the system is equally well described by this expression:

coin; (candy; System [coin, candy]

[] i; System [coin, candy] )

The first alternative in the choice subexpression represents the "normal" behaviour expected by the client. The second alternative is the Devil's one, where $\mathbf{i}$ models the hidden interaction between the Devil and the machine on action candy'. Although this interaction is invisible, we cannot drop it from our expression without affecting the behaviour of the system. If we write:

coin; (candy; System [coin, candy] [] System [coin, candy]

we are describing a system where the client can choose between getting his candy and inserting a new coin. In the original description, on the contrary, the occurrence of $i$ is not at the client's discretion; it may simply happen, unnoticeable, and the client is confronted afterwards with only one possible course of action, viz. System.

The case of "asymmetric" nondeterminism with a choice between an observable and an unobservable action as was just discussed, is often found in an OSI context. Typically we have:

normal-course-of-action

[] i; disconnect indication; ...

where a process may be forced to accept a disconnect indication although, in principle, other alternatives exist.

Table 2

Portion of the Manager of a Transport Service.

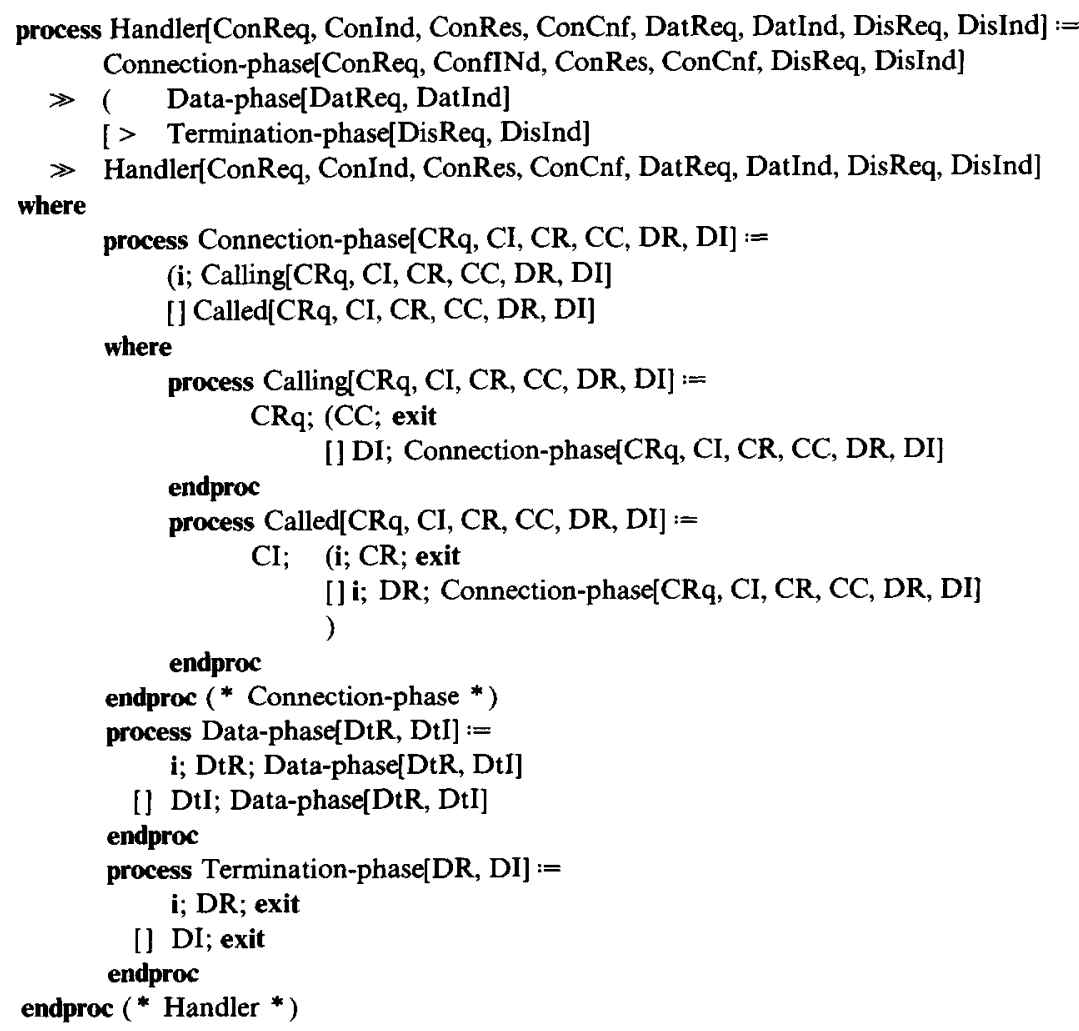




\subsection{An Example in Basic LOTOS}

In all OSI protocol specifications one can identify parts that are responsible for the management of the connections in the underlying service, i.e. the setting up, using and disconnection of the logical communication channels that exist between the service users. Here we present in Table 2 a small and simplified portion of the manager of a Transport Service, which would typically be a part of a Session Protocol. We do not discuss the Transport Service here, the uninitiated reader is referred to [44] for more information.

\section{Behavioural Equivalences}

One can describe systems at various levels of abstraction; for example it is possible to describe how they are structured internally in terms of predefined subcomponents, or how they behave from the point of view of a user or of an external observer. In moving within this range of descriptive levels, it is common to distinguish between: specifications, which are rather high level descriptions of the desired behaviour of the system, e.g. as seen by the user (extensional description); implementations, which are more detailed descriptions of how the system works or of how it is constructed starting from simpler components (intensional description).

LOTOS is a specification language which allows the specification of systems at different descriptive levels. In LOTOS the words 'specification' and 'implementation' have a relative meaning, not an absolute one. Given two (syntactically homogeneous) LOTOS specifications S1 and $\mathbf{S} 2$, we will say that $\mathbf{S} 2$ is an implementation of the specification $\mathrm{S} 1$ when, informally, S2 gives a more structured and detailed description of the system specified in S1. Structure in a LOTOS specification is another concept which cannot be given an absolute measure. We might say that a specification is made structured by a "generous" use of the parallel, the enable, and, perhaps, the disable operators, and of process definitions. For example, process 'duplex-buffer' in Section 2.4 shows more structure than its version in Section 2.3 .

The relationships between different LOTOS descriptions of a given system and, in particular, between specifications and implementations, can be studied by using a notion of equivalence, proposed in [37] and used for a CCS-like calculus in [34]. This equivalence, known as observational equivalence, is based on the idea that the behaviour of a system is determined by the way it interacts with external observers. Theories of equivalences turn out to be very useful. In fact, they allow one not only to prove that an implementation is correct with respect to a given specification but also to replace complex subsystems with simpler, equivalent ones, within a large system, thus simplifying the analysis of the latter.

A typical example of two different descriptive levels found in the OSI architecture is provided by the concepts of protocol and service [24,39]. The specification of the $\mathrm{N}$-service is implemented by the composition of the $\mathrm{N}$-protocol entities with the $(\mathrm{N}-1)$-service, and it seems natural to require that the two descriptions be equivalent. Unfortunately the complexity of OSI services and protocols is such that a proof of equivalence will certainly require the assistance of automated tools; and when the full language is used (this is of course the case for applications to OSI) the development of verification algorithms is a challenging task in itself. We are not concerned about analytical tools here. For our illustrative purposes it will be enough to give an example of a specification/implementation pair for which the equivalence proof can be carried out by hand. But before doing this, we want to stress the importance of equivalences from a slightly different perspective, namely for having a satisfactory definition of the formal semantics of the language. This will also give us the opportunity to shift our discussion of equivalences into the domain of trees, with the obvious pictorial advantages.

What is the meaning of the LOTOS expression below?

$$
\text { a; (b; stop [ ] i; c; stop) [] a; c; stop }
$$

We might apply the operational semantics of Section 2 for deriving the action tree of Fig. 8a from the expression, and then be tempted to say that the tree is the semantics of the expression. Since we regard the tree as a description of a behaviour in terms of observable actions, we would consider the colour of the tree arcs and nodes as immaterial. Similarly, we would not object on the choice of a different ordering for the outgoing arcs 


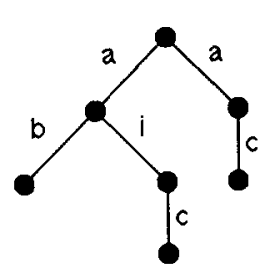

a)

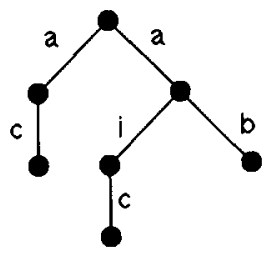

b)

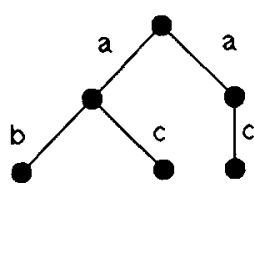

c)

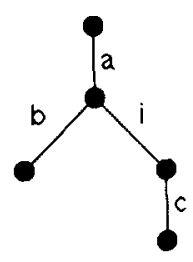

d)

Fig. 8. Comparing action trees.

of a node. For instance, we could accept the tree in Fig. $8 \mathrm{~b}$ as well. On the other hand, expression 'a; c; stop [] a; (b; stop [] i; c; stop)' also admits tree a), or tree b), as an action tree, and we may conclude that the two expressions should also be considered as equivalent. Rather than viewing the semantics of an expression as a tree, we will talk then about equivalence classes of trees and, consequently, of expressions. Once a proper notion of equivalence between trees is chosen, we will say that two expressions are equivalent (or that they have the same meaning) if their trees are in the same equivalence class. Thus the meaning of an expression can be identified with its equivalence class. We concentrate now on trees.

We have easily accepted the equivalence between trees (a) and (b) in Fig. 8. Following the discussion on nondeterminism and internal actions in Section 2.9, we would not put tree (c), where an i-action has been dropped, in the same class as (a) and (b). Consider now trees (a) and (d): do they represent the same observable behaviour? In order to give a convincing answer we need a formal definition of observational equivalence.

The idea of observational equivalence is that two systems are considered as equivalent whenever we cannot tell them apart by external observations. As external observers we do not directly see trees (a) and (d) as in Fig. 8, but we may only experiment with the keys of the two proto-pianolas in Fig. 9, which incorporate these two trees as their hidden scores. As discussed in Section 1, observations consist in simply pressing keys, one at a time, and noticing whether they are free or blocked. Our experiments are formalized by the observable sequence relation ' $\Rightarrow$ '. We refer to the notational conventions fixed in Section 2.3. However we may now imagine that $B, B^{\prime}$ and $B_{i}$ denote simply tree nodes, or states, rather than behaviour expressions (recall that behaviour expressions are node labels in transition trees). An element of the observable sequence relation is a triple $\left(B, s, B^{\prime}\right)$, where $s$ is a string of observable actions, and is written $B=s \Rightarrow B^{\prime}$. The purpose of this relation is to abstract from the invisible actions that are on the path between two tree nodes.

Definition 3.1. i) Let s denote a string $\mu_{1}^{+} \mu_{2}^{+} \ldots \mu_{n}^{+}$ of actions. We define relation $-\mathrm{s} \rightarrow$ as the obvious extension of the transition relation (tree arcs) to action sequences:

$B-s \rightarrow B^{\prime}$ if and only if there exist $B_{i}, 0 \leq i \leq n$, such that $\mathrm{B}=\mathrm{B}_{0}-\mu_{1}^{+} \rightarrow \mathrm{B}_{1} \ldots \mathrm{B}_{\mathrm{n}-1}-\mu_{\mathrm{n}}^{+} \rightarrow \mathrm{B}_{\mathrm{n}}=$ $B^{\prime}$. In particular, for $n=0$ we have $B-\epsilon \rightarrow B$ for any $B$, where $\epsilon$ is the empty string.

ii) Let $s$ denote now a string $g_{1}^{+} g_{2}^{+} \ldots g_{n}^{+}$of observable actions, and let $\mathrm{i}^{\mathrm{k}}$ denote a sequence of $k(k \geq 0)$ i-actions. Then we have $B=s \rightarrow B^{\prime}$
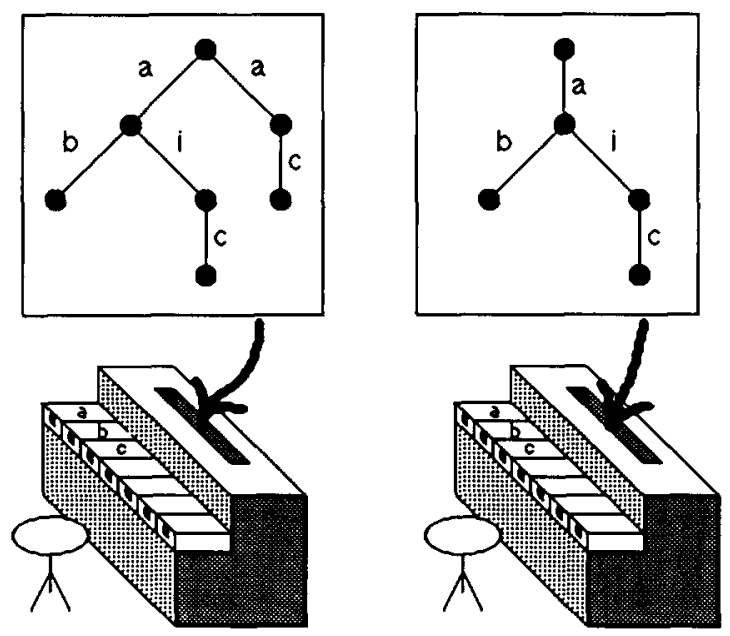

Fig. 9. Are these two proto-pianolas observationally equivalent? 
whenever there exists a sequence $\left(\mathrm{i}^{\mathrm{k} 0} \mathrm{~g}_{1}^{+} \mathrm{i}^{\mathrm{kl}} \mathrm{g}_{2}^{+} \ldots\right.$ $\left.\mathrm{g}_{\mathrm{n}}^{+} \mathrm{i}^{\mathrm{kn}}\right)$ of actions such that:

$B-\left(\mathrm{i}^{\mathrm{k} 0} \mathrm{~g}_{1}^{+} \mathrm{i}^{\mathrm{k} 1} \mathrm{~g}_{2}^{+} \ldots \mathrm{g}_{\mathrm{n}}^{+} \mathrm{i}^{\mathrm{kn}}\right) \rightarrow \mathrm{B}^{\prime}$.

This implies that $B=\epsilon \Rightarrow B^{\prime}$ whenever $B-i^{k} \rightarrow$ $B^{\prime}$, and that $B=\epsilon \Rightarrow B$ for any $B$.

Examples: given a tree path $\mathrm{B}_{0}-\mathrm{i} \rightarrow \mathrm{B}_{1}-\mathrm{a} \rightarrow \mathrm{B}_{2}$ $-\mathbf{i} \rightarrow \mathrm{B}_{3}-\mathrm{b} \rightarrow \mathrm{B}_{4}$, we may write:

$\mathrm{B}_{0}-\mathrm{iaib} \rightarrow \mathrm{B}_{4}$

$\mathrm{B}_{0}=\mathrm{ab} \Rightarrow \mathrm{B}_{4}$

$\mathrm{B}_{1}=\mathrm{a} \Rightarrow \mathrm{B}_{2}$

$\mathrm{B}_{0}=\epsilon \Rightarrow \mathrm{B}_{1}$

$\mathrm{B}_{0}=\epsilon \Rightarrow \mathrm{B}_{0}$.

Based on the observable sequence relation, we define a notion of bisimulation.

Definition 3.2. A relation $\Re$ between tree nodes is a bisimulation if for any pair $\left(\mathrm{B}_{1}, \mathrm{~B}_{2}\right)$ in $\Re$ and for any string $s$ of observable actions:

i. whenever $B_{1}=s \Rightarrow B_{1}^{\prime}$ then, for some $B_{2}^{\prime}$ : $\mathrm{B}_{2}=\mathrm{s} \Rightarrow \mathrm{B}_{2}^{\prime}$ and $\mathrm{B}_{1}^{\prime} \Re \mathrm{B}_{2}^{\prime}$

ii. whenever $B_{2}=s \Rightarrow B_{2}^{\prime}$ then, for some $B_{1}^{\prime}$ : $\mathrm{B}_{1}=\mathrm{s} \Rightarrow \mathrm{B}_{1}^{\prime}$ and $\mathrm{B}_{1}^{\prime} \mathfrak{R} \mathrm{B}_{2}^{\prime}$.

The idea of bisimulation is that two bisimilar nodes must be able to "simulate" each other, in terms of observable sequences, and then reach still bisimilar nodes. Finally:

Definition 3.3. Two tree nodes $B_{1}$ and $B_{2}$ are observationally equivalent, written $\mathrm{B}_{1} \approx \mathrm{B}_{2}$, if there exists a bisimulation $\mathfrak{R}$ which contains the pair $\left(\mathrm{B}_{1}, \mathrm{~B}_{2}\right)$.

When we talk about the observational equivalence of two trees we refer, of course, to the

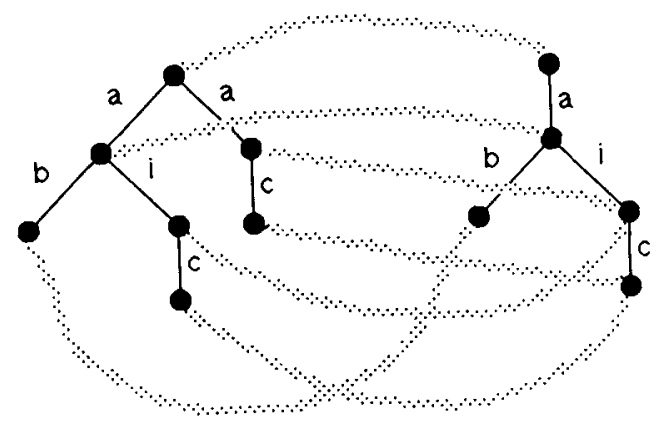

Fig. 10. A bisimulation. equivalence of their roots. For proving the observational equivalence of trees (a) and (d) in Fig. 8 , we must then provide a bisimulation between their nodes, which include also the pair of roots. The reader may check that the relation defined by the dashed lines in Fig. 10 is in fact a bisimulation: any pair of nodes connected by a dashed line satisfies conditions (i) and (ii) in Definition 3.2. Hence the two trees are observationally equivalent, and we can write:

$$
\begin{aligned}
& \text { a; (b; stop [] i; c; stop) [] a; c; stop } \\
& \quad \approx a ;(b ; \operatorname{stop}[] \mathbf{i} ; \mathrm{c} ; \text { stop) }
\end{aligned}
$$

Now that we have switched from trees back to expressions, we may try to solve our equivalence problems directly, by algebraic manipulations of the given expressions. In particular we might want to substitute some subexpression $F$ of a given expression $\mathrm{E}$ with an expression $\mathrm{F}^{\prime}$ equivalent to $\mathrm{F}$, without affecting the overall behaviour, that is, without leaving the equivalence class of $\mathrm{E}$. Unfortunately, observational equivalence is not a substitutive relation. We need to consider a different relation, called observational congruence, written ' $\approx$ ',

We will not formally define observational congruence here (see [33]). It will only suffice to say that it is defined in terms of observational equivalence, it is stronger than it (that is: $\mathrm{B} 1 \approx^{\mathrm{c}} \mathrm{B} 2$ implies $B 1 \approx B 2$ ), it is substitutive, and it satisfies a number of useful laws [21]. Three observational congruence laws are given in Fig. 11, in tree form. Recall that, by our conventions, $\mu^{+}$denotes any action.

Notice that the third law matches trees (a) and (d) of Fig. 8, thus providing another proof of their observational equivalence. We will now use the first two laws to provide the proof of observational equivalence between two systems. Consider the following basic LOTOS processes:

Process Max3-Spec [in1, in2, in3, out] :=

in 1; ( in 2, in 3, out, stop

[] in3, in2, out, stop)

[] in2; ( in1, in 3, out, stop

[] in 3 , in 1, out, stop)

[] in3; ( in1, in2, out, stop

endproc

[] in2, in1, out, stop) 


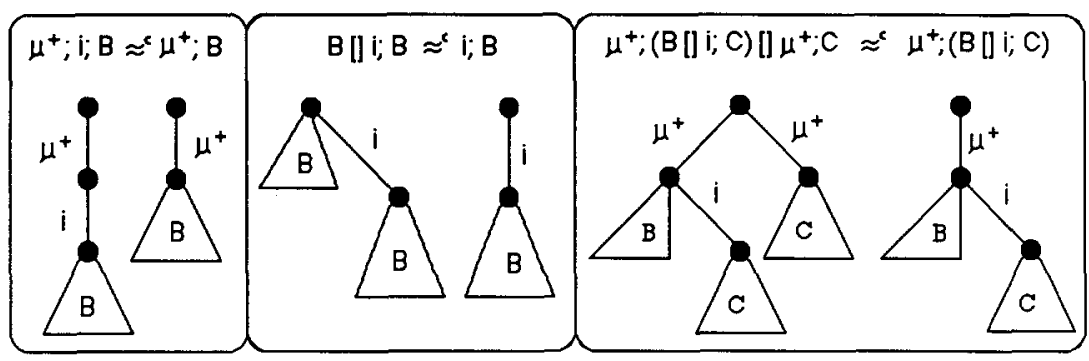

Fig. 11. Three observational congruence laws.

Process Max3 [in1, in2, in 3 , out] := hide mid in

(Max2[in1, in2, mid]

|[mid |Max2[mid, in3, out])

where

process $\operatorname{Max} 2[a, b, c]:=$

$\mathrm{a} ; \mathrm{b}$; c; stop

[]

b; a; c; stop

\section{endproc}

endproc
Process Max3 (which was already introduced in Section 2.1, Fig. 4) can be seen as an implementation of process Max3 3 Spec, in terms of process Max2. The latter describes a black box which outputs a signal only after receiving three input signals, in any order. Our claim is that Max3.Spec and Max3 are observationally equivalent. Consider the two actions trees of the processes, shown in Fig. 12.

The proof can be easily conducted by simple graphical manipulations. The first congruence law

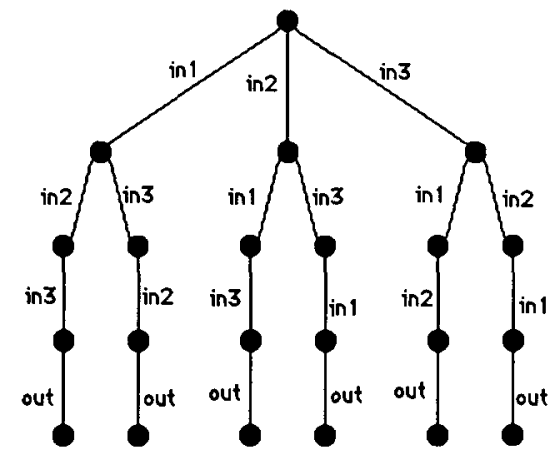

a) Action tree for Max3-spec

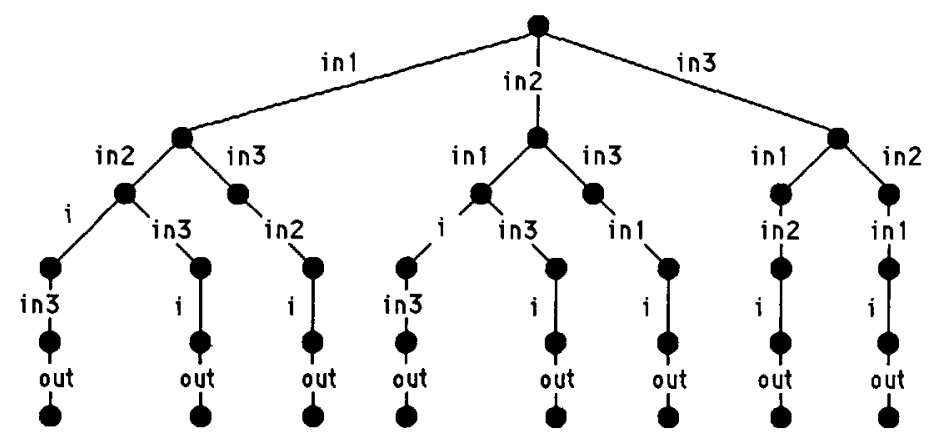

b) Action tree for Max3

Fig. 12. Two observationally equivalent action trees. 
is applied to collapse six $\mathbf{i}$-actions of the tree of Max3. Then two subtrees of the resulting tree are reduced according to the second law, and eventually the first law can be applied twice again to give us a tree identical to the one of Max3-spec. The substitution of subtrees is allowed because we work with a congruence relation. In doing this we obtain a slightly stronger result: the two trees are not only observationally equivalent, but also congruent.

A survey on observational equivalence verification algorithms can be found in [4] (see also [30]).

Apart from observational equivalence, there exist a number of other ways to compare LOTOS processes. When specifying complicated behaviours it is a generally adopted strategy to specify all the behaviour that would be acceptable in implementations of the specification. This usually leads to a specification that includes a number of options of behaviour, all of which need not necessarily be part of any single implementation. In this case, one may want to establish that the behaviour of an implementation is an acceptable reduction of the behaviour of the specification, rather than verifying their equivalence.

To deal with this question, a number of asymmetric relations between behaviours have been suggested, which all are based on the same main idea. For CSP this 'implementation relation' was introduced in [11], and for CCS in [15], which was generalized to the context of labelled transition systems in [14]. The elaboration of such a relation for LOTOS can be found in [10].

The main idea is that ' $\mathrm{B}$ red $\mathrm{S}$ ' (behaviour $\mathrm{B}$ reduces specification $S$, where $B$ and $S$ are processes) iff

i) B can only execute actions that $\mathrm{S}$ can execute; and

ii) B can only refuse actions that can be refused by $S$.

We still consider action $\mathbf{i}$ as invisible, and when we say " $B$ can execute action $x$ " we mean that $x$ is observable, and that $B=x \Rightarrow B^{\prime}$, for some process $B^{\prime}$. The key to the understanding of this relation is that a specification $S$ may be nondeterministic: after having interacted in a sequence of events $\mathrm{s}, \mathrm{S}$ may both offer and refuse a particular set $A$ of actions. Two instances of this relation are:

a) $\mathrm{B}$ red i; $\mathrm{B}$ [] i; C

b) $\mathrm{B}$ red $\mathbf{i}$; $\mathrm{B}[] \mathrm{C}$
To fix ideas, let us consider case (b). It is clear that $\mathrm{B}$ can only perform actions there are also in $\mathrm{i}$; $\mathrm{B}$ [] C, so that condition (i) is fulfilled. Also, every non-initial state of $B$ has an equivalent state in $\mathrm{i}$; $\mathrm{B}$ [] C, so that condition (ii) needs only verification for the initial state of $B$. It follows easily from $(i ; B$ [ ] C) $=\epsilon \Rightarrow B$ that all actions that can be refused by $B$ after a sequence of invisible actions can also be refused by $\mathbf{i}$; $\mathrm{B}$ [] $\mathrm{C}$ after a sequence of invisible actions.

Note that C red i; B [] C does not hold; C may refuse initial actions of $B$ that cannot be refused by i; B [] C.

The implementation relations also induce equivalences between behaviours: $\mathrm{B}$ equivalent $\mathrm{C}$ iff $\mathrm{B}$ red $\mathrm{C}$ and $\mathrm{C}$ red $\mathrm{B}$. This equivalence is referred to as failure equivalence in [11], and testing equivalence $[15,14,10]$. An advantage of these equivalences is that they do not distinguish between processes that cannot be distinguished by experiments, while this may happen with observational equivalence. As an example, consider the following processes:

$\mathrm{B}_{1}=\mathrm{a} ;(\mathrm{a} ; \mathrm{a} ; \operatorname{stop}[] \mathrm{a} ; \mathbf{s t o p})$

$\mathbf{B}_{2}=\mathbf{a} ; \mathrm{a} ; \mathbf{a} ; \mathbf{s t o p}[] \mathrm{a} ; \mathbf{a}$; stop

Both of them will certainly support the observation of action sequences $a$ and $a a$, and may or may not support the observation of $a a a$; any other observation will not be supported. In spite of this, they would be distinguished by observational equivalence, since:

$\mathrm{B}_{1}-\mathrm{a} \rightarrow(\mathrm{a} ; \mathrm{a} ; \operatorname{stop}[] \mathrm{a} ; \mathbf{s t o p})=\mathrm{B}_{3}$

and

$\mathrm{B}_{2}-\mathrm{a} \rightarrow \mathrm{a} ; \mathrm{a} ; \mathbf{s t o p}=\mathrm{B}_{4}$ and

$B_{2}-a \rightarrow a ;$ stop $=B_{5}$.

Clearly $B_{3}$ is not equivalent to $B_{4}$ because $B_{3}$ may refuse to accept the action sequence ' $a a^{\prime}$ while $B_{4}$ will certainly accept it; and $B_{3}$ is not equivalent to $B_{5}$ because $B_{3}$ may accept 'aa' while $B_{4}$ will certainly refuse it.

The name testing equivalence was chosen because in some sense this relation identifies exactly those processes that cannot be distinguished by testing. In [9] and [8] it is indicated how this relation may be further modified to support the practical testing of processes for conformance to their specification.

One of the advantages of LOTOS is that, on 
the basis of its operational semantics, different relations between specifications can be defined, which suit different needs.

\section{Data Types}

The representations of values, value expressions and data structures in LOTOS are derived from the specification language for abstract data types (ADT) ACT ONE [16]. The choice of abstract data types for LOTOS, as opposed to concrete data types, is consistent with the requirement of abstraction from implementation details which has been a guiding principle also in the design of the other component of the language (process definitions). A concrete data type implies a description of how data values are represented in memory, and how some associated procedures operate on them. In other words the data type is defined by explicitly giving its implementation. For example a Pascal queue can be defined as a list of records and a pair of procedures which manipulate it to realize the 'Add' and 'Remove' operations. An abstract data type can be seen as the formal specification of a class of concrete data types. It does not indicate how data values are actually represented and manipulated in memory, but only defines the essential properties of data and operations that any correct implementation (concrete data type) is required to satisfy. Ultimately, an ADT definition identifies a mathematical object, namely an algebra, formed by sets of data values, called data carriers, and a set of associated operations. The reader interested in the specification of ADT's in general may refer to [19] and [20].

ACT ONE is an algebraic specification method to write unparametrized as well as parametrized ADT specifications. ACT ONE, and thus LOTOS, includes the following features for the production of structured specifications:

1. use of a library of predefined data types;

2. extensions and combinations of already existing specifications;

3. parametrization of specifications, and actualization of parametrized specifications;

4. renaming of specifications.

The most basic form of data type specification in LOTOS consists of a signature and, possibly, a list of equations.

\subsection{Signature}

The first step in specifying a data type consists of defining names of data carriers and operations. The names of the data carriers are referred to as sorts. The declaration of every operation will include its domain, which consists of a list of zero or more sorts, and range, which consists of exactly one sort. The sorts and operations of a data type are referred to as the signature of that data type.

Below we list a type definition of the natural numbers, which only consists of a signature. The definition is named 'Nat_numbers', so that it may be referred to by other definitions, and combined with them. The signature of Nat_numbers consists of the single sort 'nat', and the operations ' 0 ' and 'succ'. Operation 'succ' can be applied to a single element of sort 'nat', and yields also an element of 'nat' as a result, as indicated by the notation 'nat $\rightarrow$ nat'. Operation ' 0 ' is an operation that has no arguments, yet it yields an element of 'nat', as indicated by the notation ' $\rightarrow$ nat'.

type Nat_numbers is

sorts nat

opns $0:$ : nat

succ : nat $\rightarrow$ nat

endtype

We express the fact that an operation has $n$ arguments by saying that it is an $n$-ary operation. Thus 'succ' is a unary operation, while ' 0 ' is a nullary operation. Nullary operations are called constants.

An additional example of a complete data type definition that consists only of a signature is the definition of a set of characters $\left\{a_{1}, \ldots, a_{n}\right\}$, where each character is defined as a constant:

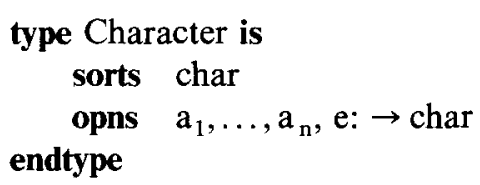

Note that there is a special symbol ' $e$ ' which is used in the next chapter to represent an error that is of sort 'char'.

The signature of a type gives all the information required to build syntactically correct terms, or value expressions, which represent data values of (some sort of) that type. A term is the result of applying an $\mathrm{n}$-ary operation to $\mathrm{n}$ terms. In particular, a constant is clearly a term. More precisely, if 
a signature contains the constant declaration:

$c: \rightarrow s$

where $\mathrm{s}$ is some sort, then we say that $\mathrm{c}$ is a constant (or a term) of sort $s$. Similarly, if an operation is declared as:

op: $s_{1}, \ldots, s_{n} \rightarrow s$

then $o p\left(t_{1}, \ldots, t_{n}\right)$ is a term of sort $s$, or an s-term, for short, where $t_{i}$ is an $s_{i}$-term, for $i=1, \ldots, n$.

For example, given the signature of type Nat numbers above, we may construct the following terms, all of sort nat:

$0, \operatorname{succ}(0), \operatorname{succ}(\operatorname{succ}(0)), \ldots$

which are meant to denote, respectively, the elements $0,1,2 \ldots$ of the algebra of natural numbers.

\subsection{Equations}

Suppose now that we want to define a 'plus' operation, which combines two nat-terms into a new nat-term:

$+_{-}:$nat, nat $\rightarrow$ nat.

The two underscore symbols ' -' mark the position of the operands with respect of the operator, which is thus defined as an infix operator. We have now the possibility to write new nat-terms, such as ' $0+\operatorname{succ}(0)$ '. To interpret these nat-terms correctly, we need a new construct to express properties of operations. This construct is the equation. The purpose of an equation is to state that two syntactically different terms denote the same value. For instance, we want to express the fact that terms ' $\operatorname{succ}(0)$ ' and ' $\operatorname{succ}(0)+0$ ' denote the same value, or, more generally, that for any nat-term $\mathrm{x}$, terms ' $\mathrm{x}$ ' and ' $\mathrm{x}+0$ ' denote the same value. A correct definition of the properties of the ' + ' operator is:

eqns

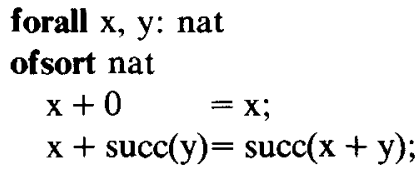

where the equations identify nat-terms (ofsort nat), and are valid whenever variables $\mathrm{x}$ and $\mathrm{y}$ are replaced by any pair of nat-terms (forall $x, y$; nat). The first equation expresses the behaviour of the plus operator when it is combined with the constant ' 0 '. The addition with a non-zero number is covered by the second equation (note that term
' $\operatorname{succ}(x)$ ' always denotes a non-zero number). By induction on the structure of terms, and by using these equations, it can be easily proved that any term containing one or more plus operations is equal to a term containing only ' 0 ' and 'succ'. This means that by introducing the plus operator we have not introduced terms that denote 'new' values which could not be expressed before. In this case, we say that the equations of ' + ' are complete w.r.t. the definition of ' $\mathrm{Nat}$ numbers'.

The specification of the natural numbers extended with the plus operation is:

type Extended_nat_numbers is

sorts nat

opns $0 \quad: \rightarrow$ nat

suce $\quad:$ nat $\rightarrow$ nat

$+_{-} \quad$ : nat, nat $\rightarrow$ nat.

eqns

forall $x, y$ : nat

ofsort nat

$$
\begin{array}{ll}
x+0 & =x ; \\
x+\operatorname{succ}(y) & =\operatorname{succ}(x+y)
\end{array}
$$

endtype

\subsection{Extensions and Combinations of Type Specifi- cations}

To specify data types with a large number of operations we need language constructs to combine already existing specifications, and/or to extend them by adding further sorts, operations and equations. This way bulky specification can be given in a stepwise fashion, and a same, simple data type can be used as a basis for several, more complex definitions.

As an example of enrichment of a type, we re-define the type Extended nat_numbers on the basis of type NaturalNumbers (both definitions are given in the previous section):

type Extended_nat_numbers is Nat_numbers

opns _ $+_{-}$: nat, nat $\rightarrow$ nat.

eqns

forall $x, y$ : nat

ofsort nat

$$
\begin{array}{ll}
x+0 & =x ; \\
x+\operatorname{succ}(y) & =\operatorname{succ}(x+y) ;
\end{array}
$$

\section{endtype}

In 'Extended_nat_numbers' we have imported the whole definition 'Nat_numbers' by referenc- 
ing it in the heading of the former, and we have enriched it with one operation and two equations. In general we may combine several type definitions, and then add specific new elements:

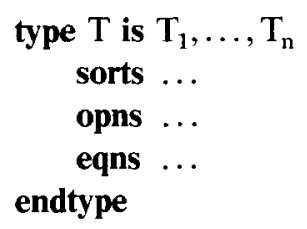

\subsection{Parameterized Types}

Parameterized data type specifications can be considered as partial specifications where only some general features of the type are described, and 'holes' are left to be filled later with further details. A queue, for instance, can be described as a parameterized type, which can later be actualized to become a queue of integers or a queue of characters.

In the absence of the parameterization features, we could define the queue of natural numbers and the queue of characters as respective enrichments of the types Nat_numbers and Characters:

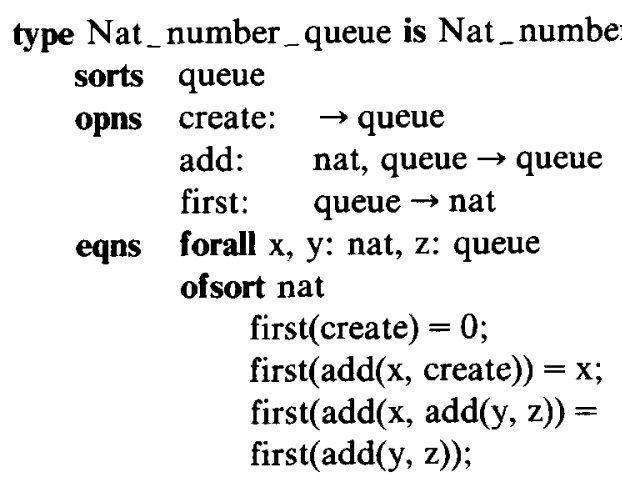

endtype

type Character_queue is Characters

sorts queue

opns create: $\rightarrow$ queue

add: $\quad$ char, queue $\rightarrow$ queue

first: $\quad$ queue $\rightarrow$ char

eqns forall $\mathrm{x}, \mathrm{y}$ : char, $\mathrm{z}$ : queue

ofsort char

first $($ create $)=e$;

first $(\operatorname{add}(x$, create $))=x$;

first $(\operatorname{add}(x, \operatorname{add}(y, z)))=$

first(add $(y, z))$;

endtype
In these new types the enrichment consists of a new sort 'queue; and of two new operations 'first' and 'add'. 'First' produces the first element at one end of the queue, and 'add' appends an element at the other end of it. The constants ' 0 ' and ' $e$ ' were already introduced respectively in the type definitions 'Nat_numbers' and 'Characters'. They are used to indicate an error when the 'first' operation is applied to an empty queue. It is clear that the two definitions above are almost the same. To avoid such duplication, we can make the sub-type of the queue that is variable a formal part of a parameterized type specification. Thus we specify a queue of a generic element, and the type of this element is made formal:

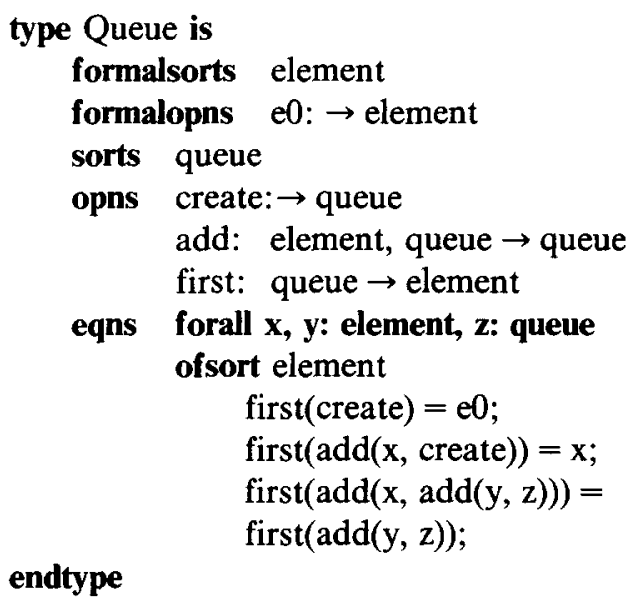

The queue is now equipped with formal components 'element' (a sort) and ' $\mathrm{e} 0$ ' (a constant), which can be actualized by the 'NaturalNumbers' or 'Characters' as follows:

type $\mathrm{Nat}_{-}$number_queue is

Queue actualizedby Nat_numbers using sortnames nat for element opnnames 0 for $\mathrm{e} 0$

endtype type Character_queue is

Queue actualizedby Characters using sortnames char for element opnnames e for $\mathrm{e} 0$

endtype

The formal part of a type definition can even contain formal equations, which are interpreted as requirements that must be fulfilled by an actual type that is substituted for it. For example we could have defined: 
type Extra_queue is

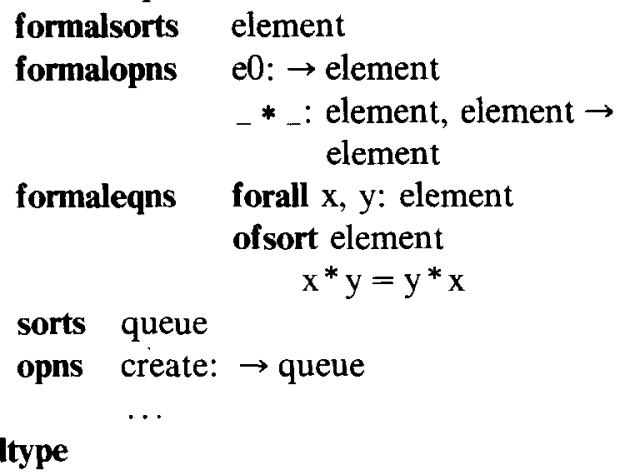

This time we could actualize 'Extra 'Enriched_nat', using the ' + ' operator for '*', but not with Characters.

\subsection{Type Renaming}

Renaming of data type specifications is useful during the development of a specification in the case where an already defined data type is needed in a specific environment, but without any changes in the intended semantics. Therefore, renaming may be done explicitly by rewriting the data type definition with new sorts and operations. Changes in the signature imply changes in the declaration of variables and in equations. Especially for long definitions this can be a cumbersome task.

The renaming operation avoids this drawback. Let us assume that the data type definition 'Queue' of the previous section is to be used in the OSI transport service environment, which deals with channels and objects to be transferred. Then the definition 'Queue' can be conveniently renamed as follows:

\section{type Connection is \\ Queue renamedby \\ sortnames channel for queue object for element \\ opnnames send for add \\ receive for first}

endtype

\section{Full LOTOS}

In Section 2 we have presented the main features of LOTOS by illustrating a subset of the language based on a finite alphabet of events. Here we increase the expressive power of basic
LOTOS by givirg a finer structure to observable actions, thus to process interactions, using the facilities for the description of data structures and values presented in Section 4. As a major advantage, in full LOTOS we will be able to enrich synchronizations with value passing, thus providing interprocess communication.

While in basic LOTOS an observable action coincides with a gate name, in full LOTOS (or, simply, LOTOS) it is formed by a gate name followed by a list of zero or more values offered at that gate: $g\left\langle v_{1} \ldots v_{n}\right\rangle$. For example:

g〈TRUE, "tree", 3$\rangle$

is the observable action offering the boolean value TRUE, character string "tree", and natural number 3 at gate $g$. Since the offered values may range over infinite sets (e.g. the natural numbers), an infinite number of observable actions is expressible in full LOTOS.

We have given the operational semantics of basic LOTOS in Section 2 with the purpose to formally define the transition relation ' $\rightarrow$ '. The axioms and inference rules for full LOTOS are meant to achieve the same goal, except that a transition may now have the form:

$\mathrm{B}_{1}-\mathrm{g}\left\langle\mathrm{v}_{1} \ldots \mathrm{v}_{\mathrm{n}}\right\rangle \rightarrow \mathrm{B}_{2}$

that is, it may involve structured, observable actions. Here we will not insist in using a formal style of presentation however, in order to avoid the introduction of further notational complexity (the complete set of axioms and inference rules for LOTOS is found in [27]).

The integration of type definitions and process definitions in a full LOTOS specification is illustrated in Fig. 13, which shows the syntax of a typical specification and a typical process definition.

Process and type definitions may appear in the where clause of a specification or process definition, in either order or even interleaved. It clearly appears that a specification and a process definition have a similar structure. A minor difference is that the behaviour expression is preceded by the keyword behaviour in the first case, and by the definition symbol ' $:=$ ' in the second case. A more significative difference is that some type definitions may appear before the behaviour expression of a specification, whereas this is not allowed in a process definition. Such type definitions are meant to 


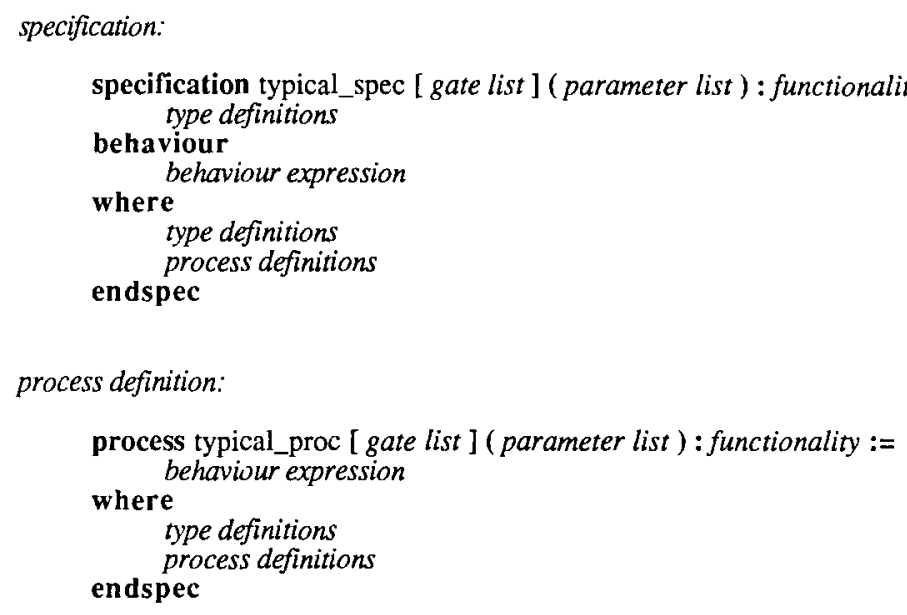

Fig. 13. Typical structures of specification and process definition.

be global definitions, which can be referenced in the parameter list of the specification and, potentially, by the environment where the specification is set to operate.

The inclusion of type definitions in specifications, and thus the possibility to express data values, are used to enrich the language in five different aspects. Values can be:

1) offered at gates, and exchanged among processes (enrichment of the action prefix operator);

2) used to express conditions to be satisfied for a given behaviour to take place (introduction of the new construct of guarding, and enrichment of action prefix with selection predicates);

3) used to generalize the choice operator.

4) used to instantiate parametric process definitions or actualize parametric behaviour expressions (parametric process definition and instantiation, 'let' construct);

5) passed by a successfully terminating process to a subsequent, enabled process (enrichment of successful termination and enabling operators); For everyone of the five features above we have indicated the corresponding constructs of the language which are affected, or newly introduced. We will address them one-by-one in the sequel.

\subsection{Value Offers and Interprocess Communication}

In formally describing formal languages it is very important to clearly distinguish between the linguistic and the meta-linguistic levels. Going back to basic LOTOS for a moment, consider the following transition of an action prefix expression:

$\mathrm{a} ; \mathrm{B}-\mathrm{a} \rightarrow \mathrm{B}$

It is clear that the first occurrence of $a$ is an actual syntactic element of the language, while the second one pertains to the meta-linguistic level used to formally describe the semantics of LOTOS expressions. Similarly, the notation ' $\mathrm{g}\left\langle\mathrm{v}_{1} \ldots \mathrm{g}_{\mathrm{n}}\right\rangle$ ' used for structured actions has only meta-linguistic value, and does not belong to the actual LOTOS syntax. We show now how the syntax of the (observable) action prefix operator is enriched in order to express such structured, observable actions. Since the structure of observable action prefix expression is:

action denotation; behaviour expression

(where the semicolon is a terminal symbol), we will concentrate on (observable) action denotation. The general structure for this construct is:

$g \alpha_{1} \alpha_{2} \ldots \alpha_{n}$

where $g$ is a gate name and the $\alpha$ 's represent a finite list of attributes. Two types of attribute are possible: a value declaration and a variable declaration.

\subsubsection{Value Declarations}

A value declaration has the form '! $\mathrm{E}$ ', where $\mathrm{E}$ is a value expression, i.e. a LOTOS expression describing a data value. Examples of value declarations are: 
!(3+5), ! $(x+1)$, :TRUE, !'example',

$$
\operatorname{mot}(\mathrm{x}), \min (\mathrm{x}, \mathrm{y}) \text {. }
$$

If we combine a value declaration attribute with a gate name $g$, and its value expression describes the value $\mathrm{v}$, then the action denotation describes action $\mathrm{g}\langle\mathrm{v}\rangle$. For example, tsap! $(3+5)$ describes action tsap $\langle 8\rangle$. If the value expression contains variables, then for each set of actual values for those variables an action is described. For example, if $x=3$ and $y=5$, then $g ! \min (x, y)$ describes event $g\langle 3\rangle$. The binding of variables to values is determined by the context, as explained below. In conclusion, if $\mathrm{E}$ is a value expression, and $\mathrm{B}$ is a behaviour expression, then the action prefix expression ' $\mathrm{g}$ ! $\mathrm{E}$; B' may offer the value of $E$ at gate $g$ and transform into $\mathrm{B}$ :

$\mathrm{g} ! \mathrm{E} ; \mathrm{B}-\mathrm{g}\langle$ value $(\mathrm{E})\rangle \rightarrow \mathrm{B}$

\section{Example}

c!largest $(0,3) ;$ stop $-\mathrm{c}\langle 3\rangle \rightarrow$ stop

\subsubsection{Variable Declarations}

A variable declaration has the form '? $\mathrm{x}$ : $\mathrm{t}$ ', where $\mathrm{x}$ is a name of a variable and $\mathrm{t}$ is its sort identifier. As was explained in Section 4, the sort identifier indicates the domain of values over which $\mathrm{x}$ ranges. Examples of variable declarations are

?x : integer, ?text : string, ?x : nat, ?active : boolean.

If a gate name is attributed with a variable declaration ' $\mathrm{x}: \mathrm{t}$ ', then action denotation ' $\mathrm{g}$ ? $\mathrm{x}: \mathrm{t}$ ' describes a set of actions, viz. the set of all actions $g\langle v\rangle$ for all values $v$ in the value domain of sort $t$. Thus, for example, 'a ? $\mathrm{x}$ : nat' describes the set of actions $\{a\langle n\rangle \mid n \in \mathbb{N}\}$. Everyone of these actions is the label of a possible transition of the transition tree. The effect of a transition in this ase is slightly more complicated than for the case of value declaration. Consider the action prefix behaviour expression ' $\mathrm{g}$ ? $\mathrm{x}: \mathrm{t} ; \mathrm{B}(\mathrm{x})$ ', where $\mathrm{B}(\mathrm{x})$ denotes a behaviour expression parameterized by some variable $\mathrm{x}$ occurring in some value expression. Then the associated transitions are:

$\mathrm{g}$ ? $\mathrm{x} ; \mathrm{t} ; \mathrm{B}(\mathrm{x})-\mathrm{g}\langle\mathrm{v}\rangle \rightarrow \mathrm{B}(\mathrm{v})$

where ' $v$ ' is any value in the domain of sort $t$, and $B(v)$ indicates that after the transition has occurred, the value ' $v$ ' has been substituted for ' $x$ ' in $\mathrm{B}(\mathrm{x})$. $\mathrm{B}(\mathrm{x})$ represents the scope where the bind- ing associated with the value declaration '? $\mathrm{x}: \mathrm{t}$ ' applies. Let us clarify these concepts with an example. Consider the action prefix expression in Fig. 14.

The whole expression does not include free variables, since the occurrences of ' $x$ ' and ' $y$ ' in the value expression 'largest $(\mathrm{x}, \mathrm{y})$ ' are bound, that is, they fall within the scopes associated to their binding occurrences in the two variable declarations. However, if we consider expression ' $b$ ? $y$ : nat; c!largest $(x, y)$; stop' in isolation, then the occurrence of ' $\mathrm{x}$ ' in 'largest $(\mathrm{x}, \mathrm{y})$ ' is free, as no binding occurrence of ' $x$ ' is there any more to bind a value to it. A possible sequence of two transitions for the whole expression is:

a? : nat; b?y : nat; c!largest $(x, y) ;$ stop

$-\mathrm{a}\langle 0\rangle \rightarrow$

b?y : nat; c!largest $(0, y) ;$ stop

$-\mathrm{b}\langle 3\rangle \rightarrow$

c!largest $(0,3)$;stop

Note how variables are replaced by values, in two steps. Because of the binding of values 0 and 3 to variables $x$ and $y$, which allows the new process to refer to these values, we could say that, rather than offering values, the process offers to accept values, and think of the '?' symbol as indicating input. In contrast, the values offered via a value declaration in an action prefix expression ('g! $\mathrm{E}$; $\mathrm{B}^{\prime}$ ) may be thought of as output.

The usual rules for nested scopes apply. E.g., consider expression ' $a x$ : $t$; $b$ ? : $t$; $c$ ! $(x+1)$; stop': the value output at gate $c$ will depend on the value input at gate $b$, not gate $a$. The combination of value declarations and variable declarations in the same action denotation ' $\mathrm{g} \alpha_{1} \alpha_{2} \ldots \alpha_{\mathrm{n}}$ ' has the obvious interpretation. For example:

$$
\begin{aligned}
& \text { g1!sap1?x : cep-sort!'test'; } \mathrm{B}(\mathrm{x}) \\
& \quad-\mathrm{g} 1\langle\text { sap1, cep-3, 'test' }\rangle \rightarrow \mathrm{B}(\text { cep-3) }
\end{aligned}
$$

if cep-3 is a value of sort cep-sort.

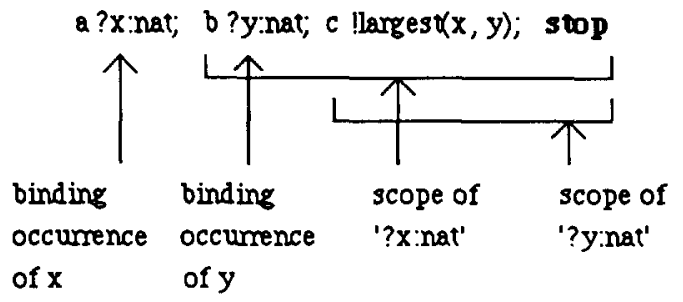

Fig. 14. Binding occurrences of variables and associates scopes. 
with successful termination, which is discussed in Section 5.5.

\subsection{Conditional Constructs}

Having added the facilities for defining and describing values in LOTOS we may now express behaviours that depend on conditions on values. Such conditions are expressed as equations that relate two value expressions: the condition is met if the two expressions evaluate to the same value, in the data type environment of that condition. Also value expressions of sort Bool of the standard data type Boolean are allowed as conditions: an expression $E$ of sort $B o o l$ is used as a shorthand for the equation $E=$ true. Below we will refer to the conditions of both kinds as predicates. By convention, predicates appear enclosed in square brackets.

\subsubsection{Selection Predicates}

An additional feature of action denotations is that of the selection predicates. An action denotation may now terminate with a predicate, containing some of the variables that occur in the variable declarations of the action denotation. Such predicate is meant to impose restrictions on the values that may be bound to these variables in synchronization events. We illustrate this by two examples.

The only possible transitions of expression 'sap?x : nat $[x>3]$; sap2! $x$; stop' are:

sap? : nat $[x<3]$; sap2! $x$; stop

$-\operatorname{sap}\langle 0\rangle \rightarrow$

sap2!0; stop

sap? $\mathrm{x}$ : nat $[\mathrm{x}<3]$; $\operatorname{sap} 2$ ! $\mathrm{x}$; stop

$-\operatorname{sap}\langle 1\rangle \rightarrow$

sap2!1; stop

sap? : nat $[\mathrm{x}<3]$; sap2! $\mathrm{x}$; stop

$-\operatorname{sap}\langle 2\rangle \rightarrow$

sap2!2; stop

In OSI applications there exist examples where two processes negotiate the value of a parameter in an interaction, each one imposing its own condition. For example, two Transport entities may negotiate the 'quality of service' of the underlying Network Service [25]. A simplified example of negotiation is given below. hide sap in

sap? $\mathrm{x}$ : nat $[\mathrm{x}<\max ]$; $\mathrm{B} 1(\mathrm{x})$

|[sap] $\mid$

sap?y : nat $[y>\min ]$; B2(y)

This process can make internal transitions to any of the processes

hide sap in

$$
\mathrm{B} 1(\mathrm{n})|[\mathrm{sap}]| \mathrm{B} 2(\mathrm{n})
$$

with ' $n$ ' in the open interval (min, max).

\subsubsection{Guarded Expressions}

Any behaviour expression may be preceded by a predicate and an arrow (that is, by a 'guard'). The interpretation is that if the predicate holds, then the behaviour described by the behaviour expression is possible, otherwise the whole expression is equivalent with stop. A typical scenario is one of a choice between several guarded expressions.

Examples:

$$
\begin{aligned}
{[\mathrm{x}>0] } & \rightarrow \text { sap! } \mathrm{x} ; \mathrm{P}[\ldots](\mathrm{x}, \ldots) \\
{[][\mathrm{x} \leq 0] } & \rightarrow \text { sap!-x; } \mathrm{P}[\ldots](\mathrm{x}, \ldots)
\end{aligned}
$$

If $x=1$ the above process is equivalent with 'sap! 1 ; $P[\ldots](1, \ldots)$. If $x=-3$, it is equivalent with 'sap!3; P[...] $(-3, \ldots)$ '. Case analysis can be specified easily, viz.

$$
\begin{aligned}
{\left[\text { cond }_{1}\right] } & \rightarrow \text { process }_{1} \\
{[]\left[\text { cond }_{2}\right] } & \rightarrow \text { process }_{2} \\
\ldots & \\
{[]\left[\text { cond }_{n}\right] } & \rightarrow \text { process }_{n}
\end{aligned}
$$

The conditions in the guards need not be exclusive, e.g.

$$
\begin{aligned}
{[x>0] } & \rightarrow \text { process }_{1} \\
{[][x=5] } & \rightarrow \text { process }_{2} \\
{[][x<9] } & \rightarrow \text { process }_{3}
\end{aligned}
$$

\subsection{Generalized Choice}

Using the choice-operator '[]' we can only express a finite number of alternatives. In general, we may want to do more. Let $\mathrm{B}(\mathrm{x})$ be a behaviour expression that may depend on a variable $\mathrm{x}$, say, of sort 'nat'. We can now specify the choice among the processes $\mathrm{B}(\mathrm{v})$ for all nat-values $\mathrm{v}$ by writing:

choice $x$ : nat [] $\mathrm{B}(\mathrm{x})$

Notice that the generalized choice construct allows 
an alternative representation for the action prefix construct, when this includes a variable declaration:

$\mathrm{a} ? \mathrm{x}: \mathrm{t} ; \mathrm{B}(\mathrm{x})$

is equivalent to

choice $x: t[]$ a! $x ; B(x)$

There are more useful applications, however:

choice $x: t$ [ ] $i$; $B(x)$

offers a nondeterministic choice between the different instances of $\mathrm{B}(\mathrm{x})$, and so does:

choice $x: t[] a ; B(x)$

where $a$ may be any action denotation. More than one variable may be used as an index, so that we may write:

choice $x_{1}: t_{1}, \ldots, x_{n}: t_{n}[] B\left(x_{1}, \ldots, x_{n}\right)$

Also, sets of gate identifiers may be used for indexing, e.g.:

choice $\mathrm{g}$ in $\left[\mathrm{a}_{1}, \ldots, \mathrm{a}_{\mathrm{n}}\right][]$ Process-X $[\mathrm{g}](\ldots)$

In this case a choice is expressed among $n$ instances of Process-X: for each one of them formal gate $g$ is actualized with a different element of the gatelist $\left[a_{1}, \ldots, a_{n}\right]$.

\subsection{Parametric Processes}

Full LOTOS offers the possibility to parameterize process definitions not only in terms of formal gates (as is the case with basic LOTOS) but also in terms of a parameter list, which is a list of variable declarations: $\mathrm{x}_{1}: \mathrm{t}_{1}, \ldots, \mathrm{x}_{\mathrm{n}}: \mathrm{t}_{\mathrm{n}}$. The syntax for process definition in full LOTOS (as anticipated in Fig. 13) is thus:

process typical_proc [ gate list] $\left(\mathrm{x}_{1}: \mathrm{t}_{1}, \ldots, \mathrm{x}_{\mathrm{n}}: \mathrm{t}_{\mathrm{n}}\right)$ : : functionality

endproc

$$
:=\ldots
$$

Also specifications can be parametric, and the syntax is extended analogously. Typically, the variables $x_{1}, \ldots, x_{n}$ occur as free variables in the behaviour expression which defines the behaviour of the process or specification. In instantiations, these variables are replaced by value expressions (which may include variables): an instantiation of the typical_proc above has the form:

typical_proc $[$ actual gate list $]\left(\mathrm{E}_{1}, \ldots, \mathrm{E}_{\mathrm{n}}\right)$
Of course it is required that expressions $E_{1}, \ldots, E_{n}$ match, one-by-one, the sorts of the variables $\mathrm{x}_{1}, \ldots, \mathrm{x}_{\mathrm{n}}$. This is similar to passing parameters to procedures or functions in traditional programming languages.

\section{Example:}

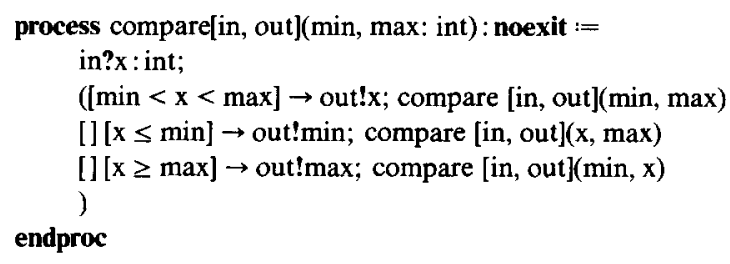

The meaning of the instantiation of a process is the behaviour expression that is obtained by substituting the actual parameters for the formal ones, avoiding naming clashes by suitable renaming of binding and bound identifiers, e.g.

compare[one, two] $(x, 2 * x)$

is equivalent with

one?y : int;

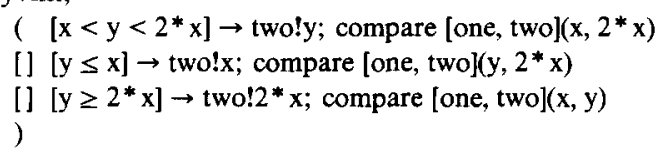

A more direct way to associate value expressions $E_{1}, \ldots, E_{n}$ to the free variables $x_{1}, \ldots, x_{n}$ of a behaviour expressions $\mathrm{B}\left(\mathrm{x}_{1}, \ldots, \mathrm{x}_{\mathrm{n}}\right)$ is offered by the 'let' construct:

let $x_{1}: t_{1}=E_{1}, \ldots, x_{n}: t_{n}=E_{n}$ in $B\left(x_{1}, \ldots, x_{n}\right)$

\subsection{Sequential Composition with Value Passing}

Having the possibility to express values it is useful and, sometimes, highly desirable to be able to pass information from the first process in a sequential composition to the second process. In the previously used example:

Connection-Phase[...] $\gg$ Data-Phase[...]

we would like to express that the behaviour of the Data-Phase depends on parameters that are established in the Connection-Phase. The DataPhase is defined as a parametric process, with such parameters as the expedited-data-option that indicates whether expedited data can be transmitted or not, and the quality-of-service that determines the quality of the connection during the Data-Phase. Therefore, we need a mechanism 
for passing these parameters from the Connection-Phase to the Data-Phase, at the moment when the former enables the latter. To be able to do such things we must generalize the notion of successful termination, and with that extend the language features with respect to sequential and parallel composition, and add some static constraints to the language.

\subsubsection{Successful Termination with Value Offers}

In basic LOTOS the exit process is used to specify the successful termination of a process. We allow now the exit process to have a finite list of value expressions added to it. The values expressed are those that are passed on to the subsequent process. Examples:

$a ? x$ : nat; b?y : nat; exit(largest $(x, y))$

tsap!cei

?quality-of-service: quality-parameter-sort

?expedited-data-option: bool

;exit(quality-of-service, expedited-data-option)

The list of the sorts of the values offered at successful termination is called the functionality of that termination. The examples above have respective functionalities $\langle$ nat $\rangle$ and $\langle q u a l i t y$-parametersort, bool $\rangle$.

In a sequential composition the number and sorts of the values that are passed at the successful termination of the first process must be known. This implies that all the (alternative) successful terminations of the first process must have the same functionality; this functionality is defined as the functionality of the first process. Some rules are needed for determining the functionality of behaviour expressions, together with some constraints on the ways expressions with different functionalities can be combined. They are listed below. (We write 'func(B)' to denote the functionality of expression B.) stop. The functionality of processes that do not terminate successfully at all, like stop, is indicated with noexit.

exit. Simple successful termination without value passing has a functionality that is indicated by the same name: func(exit) $=$ exit.

Action prefix. The functionality of an expression is clearly unaffected by the prefixing of an action denotation: func (action denotation; $\mathrm{B})=\mathrm{func}(\mathrm{B})$.

Choice. If $\mathrm{B}_{1}$ and $\mathrm{B}_{2}$ are processes that both can terminate successfully, then the functionality of the choice expression ' $B_{1}[] B_{2}$ ' can only be defined if the restriction is imposed that $B_{1}$ and $B_{2}$ have the same functionality, in which case this is the functionality of the expression. On the other hand, if func $\left(B_{1}\right)=$ noexit, or func $\left(B_{2}\right)=$ noexit, then func $\left(B_{1}[] B_{2}\right)$ is defined, respectively, as func $\left(B_{2}\right)$ and func $\left(B_{1}\right)$.

For generalized choice the rule is simple: func(choice... [] $\left.B^{\prime}\right)=\operatorname{func}\left(B^{\prime}\right)$.

Disabling. This case is analogous to that of (binary) choice:

$$
\begin{aligned}
& \operatorname{func}\left(B_{1}\right)=\operatorname{func}\left(B_{2}\right)=\operatorname{func}\left(B_{1}\left[>B_{2}\right)\right. \text {, or } \\
& \operatorname{func}\left(B_{1}\right)=\text { noexit, and } \\
& \operatorname{func}\left(B_{1}\left[>B_{2}\right)=\text { func }\left(B_{2}\right)\right. \text {, or } \\
& \text { func }\left(B_{2}\right)=\text { noexit, and } \\
& \operatorname{func}\left(B_{1}\left[>B_{2}\right)=\operatorname{func}\left(B_{1}\right)\right. \text {. }
\end{aligned}
$$

Parallel composition. In the case of parallel composition, the functionality restrictions/definitions are:

func $\left(B_{1}\right)=\operatorname{func}\left(B_{2}\right)=\operatorname{func}\left(B_{1}\right.$ op $\left.B_{2}\right)$, or

\begin{tabular}{|c|c|}
\hline $\begin{array}{l}\text { a?x: int; exit } \||| b ! ' \text { anystring'; exit } \\
\text { a?x: int; exit } \||| b ! ' \text { anystring'; stop } \\
\text { exit(3) } \| \mid \text { exit(5) } \\
\text { exit(3, any bool) } \mid \| \text { exit(any nat, true) }\end{array}$ & $\begin{array}{l}\text { has functionality exit. } \\
\text { has functionality noexit. } \\
\text { has functionality 'nat', but does not terminate successfully. } \\
\text { has functionality 'nat, bool', and terminates successfully } \\
\text { by offering value pair ( } 3 \text {, true). }\end{array}$ \\
\hline
\end{tabular}
func $\left(B_{1}\right)=$ noexit, and func $\left(B_{1}\right.$ op $\left.B_{2}\right)=$ noexit, or

func $\left(B_{2}\right)=$ noexit, and func $\left(B_{1}\right.$ op $\left.B_{2}\right)=$ noexit.

Table 4 
where 'op' is any parallel operator. Again, if $\mathrm{B}_{1}$ and $B_{2}$ are processes that terminate successfully, then we can compose them in parallel only if they have the same functionality, in which case this becomes the functionality of the parallel expression. In fact, the parallel composition of two processes only terminates successfully if both terminate with the same list of values, in which case the composition terminates also with that list. In this respect, it may be convenient to use the any-construct, as a parameter of the exit process. It has the format 'any sort-identifier', and can match any value of sort sort-identifier'. For instance, exist(any nat) is a process that can terminate successfully with the offer of any nat-value at the special gate $\delta$. It is clear that ' $\mathrm{B}_{1}$ op $\mathrm{B}_{2}$ ' cannot terminate successfully whenever one of the component processes cannot do so. Examples are given in Table 4.

The reason why there may exist a process $B$ that cannot terminate successfully, while func(B) is different from noexit, is that functionality and actual termination are two different things. The former is a sort of static typing mechanism, which is only meant to guarantee the predictability of the list of sorts offered at successful termination, in case that such termination occurs. The actual occurrence of a successful termination, in general, cannot be decided statistically, nor dynamically, since this problem is equivalent to the well-known 'Halting Problem' for Turing machines [23]. The functionality typing scheme helps in avoiding constructions however, of which the absence of successful terminations can be decided statically.

Process Definitions and Instantiations. Both a specification and a process definition include in their headers a parameter indicating the functionality of that specification or process definition, (see Fig. 13), which is defined as the functionality of the behaviour expression of that specification or process definition. In this functionality parameter a functionality ' $t_{1}, \ldots, t_{n}$ ' is combined with the keyword exit, so that the three possible formats of this parameter are:

noexit

exit

$\operatorname{exit}\left(t_{1}, \ldots, t_{n}\right)$

where $t_{1}, \ldots, t_{n}$ is a list of sorts. On the other hand, in process instantiations the functionality is not given explicitly; it is defined however, as that of the associated process definition.

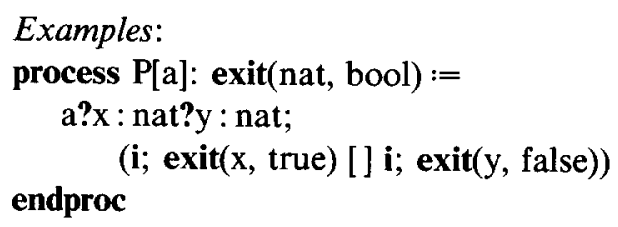

process $Q[a, b]$ : exit :=

$\mathrm{a} ? \mathrm{x}$ : nat;

endproc

(b!x; exit [] i; Q[a, b])

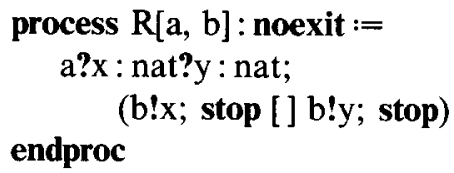

5.5.2. Accepting Values from the Enabling Process

Once an process $B_{1}$ with the desired functionality, say exit $\left(t_{1}, \ldots, t_{n}\right)$, has been defined, its sequential composition with another process $B_{2}$ has the following form:

$B_{1} \gg$ accept $x_{1}: t_{1}, \ldots, x_{n}: t_{n}$ in $B_{2}$

Here $x_{1}, \ldots, x_{n}$ are the variables used in $B_{2}$ for the $n$ values passed at the successful termination of $B_{1}$. The obvious requirement is that the functionality of $B_{1}$ be matched by the list of sorts $t_{1}, \ldots, t_{n}$ after the accept keyword. It is also clear that the functionality of the whole construct is defined as the functionality of $B_{2}$. The example quoted at the beginning of this section can now be correctly specified as follows:

$$
\begin{aligned}
& \text { Connection-Phase }[\ldots](\ldots) \\
& \text { accept } \\
& \text { quality-of-service: quality-parameter-sort } \\
& \text { expedited-data-option: bool } \\
& \text { in } \\
& \text { Data-Phase[...] } \\
& \text { (quality-of-service, expedited-data-option) }
\end{aligned}
$$

As a concluding remark we would like to observe that the value passing in sequential composition can be considered as a special case of the value passing in parallel composition. We may indeed imagine that the enabling process synchronizes its last action (successful termination) with an "accepting" action implicitly prefixed to the enabled process, and that data is passed by this 
$(*$

This is a slightly modified version of

the Daemon Game specification by W.F. Chan and K.J. Turner [13]

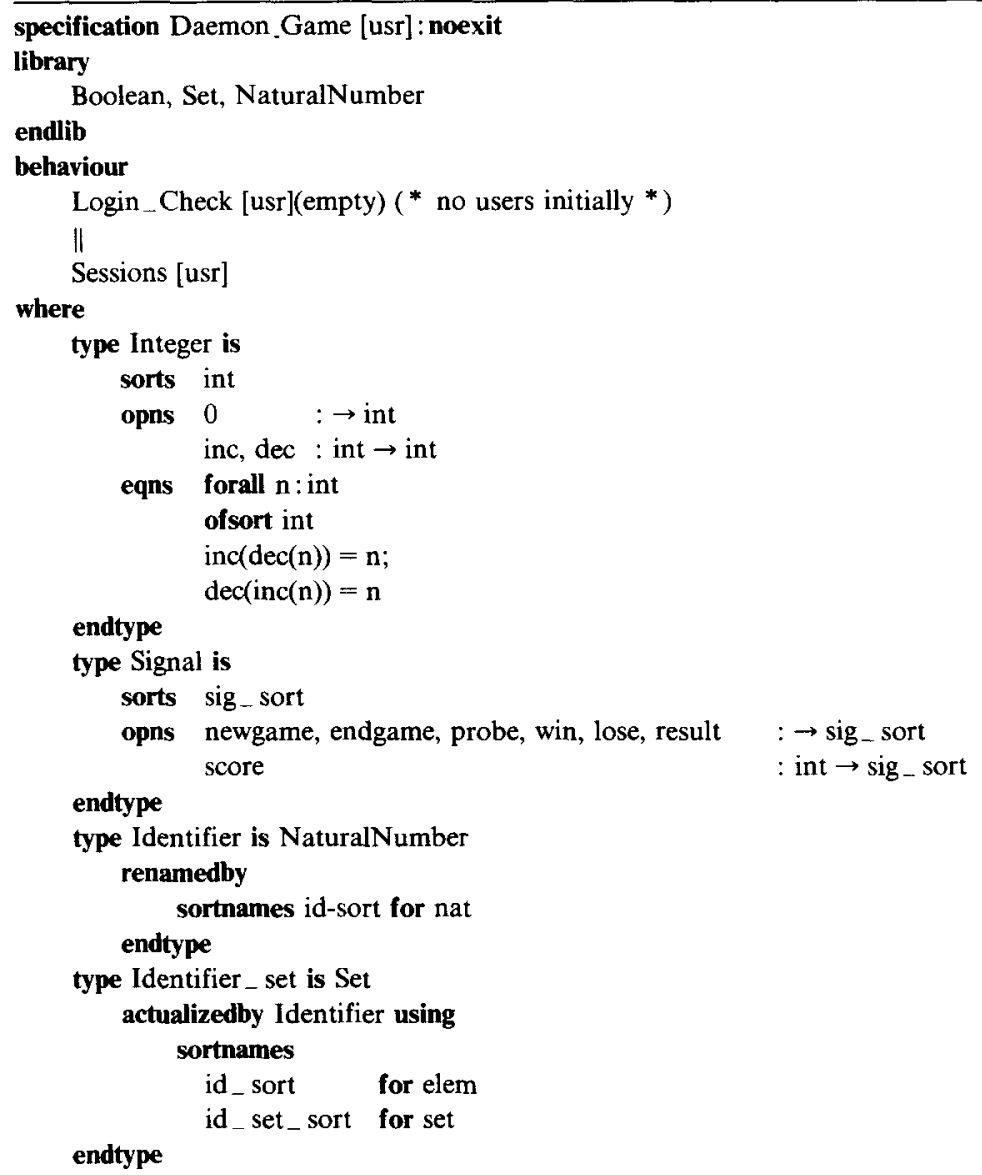

The following process ensures that users are given different identifiers on logging in. A set of identifiers in use is maintained

process Login_Check [usr](used_id_set:id_ set_ sort) : noexit :=

usr ? id: id _ sort!newgame [id NotIn used_id_ set];

Login_Check [usr] (insert (id, used_id_. set))

[] usr ?id:id_sort!endgame [id IsIn used_id_set];

Login_Check [usr] (remove (id, used_id_ set))

[] usr ?probe [id Isn used _ id _ set];

Login_Check [usr] (used _ id _ set)

[] usr ?id:id _ sort!probe [id IsIn used _ id _ set];

Login_Check [usr] (used _ id _ set)

[] usr ?id:id sort!win [id IsIn used _ id _ set];

Login_Check [usr] (used_ id _ set)

[] usr ?id:id_ sort!lose [id IsIn used_id_ set];

Login_Check [usr] (used _ id _ set)

[] usr ?id: id _ sort!result [id IsIn used id $_{-}$set];

endproc

Login_Check [usr] (used_ id _ set)

$(*$

The following process specifies the permitted sequences of interactions between the users and the game as an infinite set of processes in parallel, one for the independent behaviour of each user session

Fig. 15. 


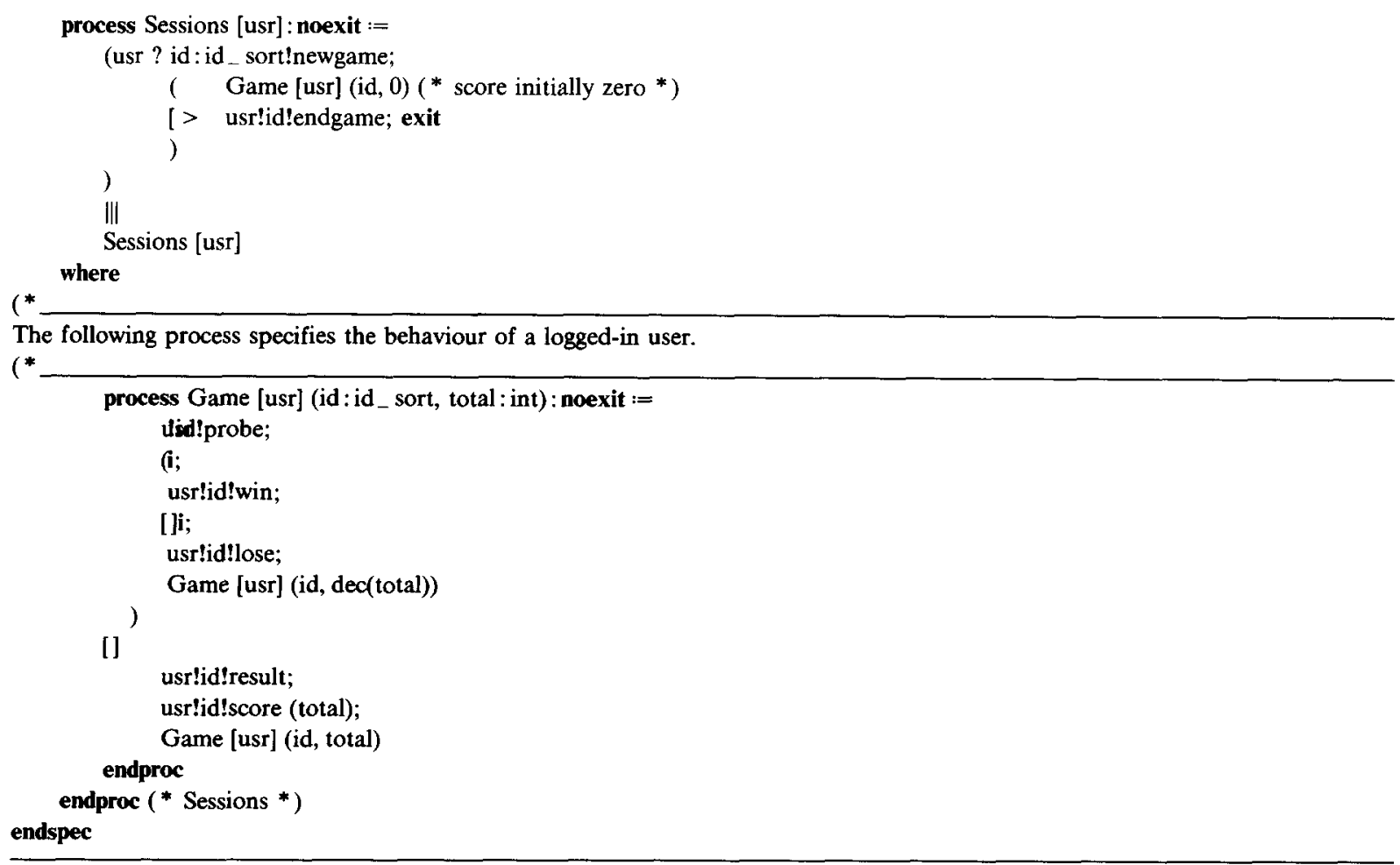

Fig. 15 (continued).

interaction. We should also regard this communication as private to the enabling and the enabled processes, that is, hidden from other processes. In fact, the operational semantics of the enabling operator in full LOTOS [27] exactly reflects this point of view.

\section{An Example of Constraint-Oriented Specifica- tion}

Structured programming, in the context of traditional programming languages, allows the programmer to take a "divide-and-conquer" approach and partition his/her task into smaller sub-tasks to be handled separately. Similarly, the constraint-oriented specification style is a "divideand-conquer" approach by which the LOTOS user conceives his/her specification as a collection of clearly separated, small pieces (processes), each one expressing few constraints on the temporal ordering of the system events. All these pieces are then composed via the parallel operator (with synchronization), which acts as a logical conjunction (AND) of all the constraints. As a consequence, any action occurring at some synchroniza- tion gate is simultaneously subject to all the constraints expressed by the processes sharing that gate. We gave a trivial example of composition of constraints in Section 2.4, in discussing the general parallel operator. We provide here (Fig. 15) a more complex example of the constraint-oriented specification style, written by Chan and Turner [13].

In the 'Daemon Game' a player may start a new game, probe the system for randomly incrementing or decrementing his score, ask for the score, and quit the game. The system may support an unlimited number of players, and every player is required to specify his own 'id' every time he/she interacts with the system. In the specification all users interact with the system via a unique gate (usr), but they are distinguished by their respective id's. All the observable actions have the unique form:

usr $\langle\mathrm{id}, \mathrm{sig}\rangle$

where id, of id sort, is the identifier of some player and sig, of sig_sort, is a signal in the set \{newgame, endgame, probe, win, lose, result $\} \cup$ Scores (a set isomorphic to the set of integers). The specification has been conceived as the com- 
position of two main concerns, embodied by processes Login_Check and Sessions. The first process does not impose any temporal constraint on actions, and is only sensitive to the actions where the signal is either 'newgame' or 'endgame', since its only concern is to properly maintain the set of user id's (Used_Id_Set). Any other action is simply absorbed. The second process (Sessions) is the interleaved composition of an infinite number of sessions, where an individual session is described by the behaviour expression at the left of the 'III' operator. A session is opened and closed when a player gives, respectively, the 'newgame' and the 'endgame' signals; the actual game is described by process 'Game'. Any individual instance of process Game is only concerned with actions characterized by a fixed value of parameter 'id', in order to properly maintain and display the score of a specific player. Processes Sessions and Game do impose some temporal constraints to the actions: for instance, winning or losing must always be preceded by probing.

\section{Conclusions}

We have presented the specification language LOTOS. The language has a strong algebraic nature and the first encounter with the apparently complex symbology of specifications may be discouraging. However, we hope we have proved, with the examples given, that once the user has achieved some familiarity with the operators of the language, he/she can specify systems in a natural way which reflects quite directly the way the system's structure and behaviour are conceived at the intuitive level. The specifier, in general, does not feel forced to express unnecessary details with respect to his/her abstract view of the processes being specified.

LOTOS has the merit (and takes the risks) of being based on relatively new and powerful theories, which so far have mainly been confined to academic environments. The wide exposure that the language is currently undergoing by its application to the specification of OSI protocols and services [42] is a valuable test for the practical applicability of those theories. The first results are encouraging: the LOTOS specifications that have been produced so far (e.g. $[1,3,12,17,40,41,43,44$, $45,47]$ and many others), indicate that such quite complex systems can be specified with an intuitively appealing structure, and be relatively concise (when compared with other formal description techniques). The conciseness and readability could be increased even further if good notational facilities are developed for the specification of data types, which now in many cases are a substantial part of a specification. Work in this direction is under development [29].

An important problem to be addressed in producing a realistically complex specification relates to the tradeoff between process and type definitions. It is a fact that many elements of a system can be specified both as processes and data types. On one hand we may rule out this problem is a mere matter of taste and style. On the other hand the interplay between processes and types has an impact also on the analysis of specifications. It is felt that a deeper understanding of the relation between the two components could be beneficial, and that some harmonization between them could be attempted (in the sense, for instance, of devising a common semantical model). This is an area where interesting developments are possible.

An important element in the eventual success of LOTOS will be the adequate training of those that are to apply it in practice [28]. The current trend of the growing importance of formal methods in computer science and telecommunications is not yet reflected in the education of many of its practitioners. This requires a coordinated effort in the development of courses and teaching material, to which this tutorial is a contribution. However, as time passes this problem will disappear. In the longer run the prospects for techniques like LOTOS are bright. Its link to a sound formal theory, and the ongoing efforts to build tools for its application to the design, analysis and testing of open distributed systems $[6,18,31,46]$ offer hopes of a future in which systems can be developed faster and with more reliability than today.

\section{Acknowledgements}

The authors gratefully acknowledge the direct and indirect contributions to this tutorial from their fellow LOTOS-eaters, with whom they have worked together during several years developing the language, applying it, and building tools for it. This work has taken place in several environ- 
ments, of which the most important are COST11 bis/TOS, ISO/TC97/SC21/WG1/FDT Subgroup $\mathrm{C}$, the ESPRIT/SEDOS Project, and the IPS Group at the University of Twente (The Netherlands). We would like to mention specifically Luigi Logrippo, Jan de Meer, Elie Najm, Giuseppe Scollo, Alastair Tocher and Chris Vissers. We would like to thank Ken Turner and W.F. Chan for their example of the Daemon Game in LOTOS, which we have included in the paper. The first author also wishes to thank Rocco De Nicola for useful discussions during the writing of parts of this paper. This work was partially supported by the CEC as part of the ESPRIT/ SEDOS project.

\section{References}

[1] I. Ajubi, "Draft Formal Specification of the OSI Connection-Oriented Session Protocol in LOTOS", ISO/TC 97/SC 21 N1486, February 1986.

[2] J. Bergstra, J.W. Klop, "Process Algebra for Synchronous Communication", Information and Control 60, pp. 109-137, 1984.

[3] F. Biemans, P. Blonk, "On the Formal Specification and Verification of CIM Architectures Using LOTOS", Computers in Industry 7, pp. 491-504, 1986.

[4] T. Bolognesi, S.A. Smolka, "Fundamental Results for the Verification of Observational Equivalence: a Survey", proceedings of the IFIP Seventh International Symposium on Protocol Specification, Testing, and Verification, $\mathrm{H}$. Rudin and C. West (eds.), North-Holland, 1987.

[5] G. Boudol, "Notes on algebraic calculi of processes, Rapport de Recherce No. 395, INRIA, Sophia Antipolis, April 1985.

[6] J.P. Briand, M.C. Fehri, L. Logrippo, A. Obaid, "Executing LOTOS Specifications", in: B. Sarikaya, G.V. Bochmann (eds.), Proceedings of IFIP Workshop 'Protocol Specification, Testing, and Verification VI', pp. 73-84, North-Holland, Amsterdam, 1987.

[7] E. Brinksma, "A Tutorial on LOTOS", in: M. Diaz (ed.), Proceedings of IFIP Workshop 'Protocol Specification, Testing, and Verification V', pp. 171-194, North-Holland, Amsterdam, 1986.

[8] E. Brinksma, "On the Existence of Canonical Testers", Memorandum INF-87-5, University of Twente, January 1987.

[9] E. Brinksma, G. Scollo, "Formal Notions of Implementation and Conformance in LOTOS", Memorandum INF87-13, University of Twente, November 1986.

[10] E. Brinksma, G. Scollo, C. Steenbergen, "LOTOS specifications, their implementations, and their tests", in: B. Sarikaya, G.V. Bochmann (eds.), Proceedings of IFIP Workshop 'Protocol Specification, Testing, and Verification VI', pp. 349-360, North-Holland, Amsterdam, 1987.

[11] S.D. Brookes, C.A.R. Hoare, A.D. Roscoe, "A Theory of
Communicating Sequential Processes", Journal of ACM, Vol. 31, No. 3, pp. 560-599, 1984.

[12] V. Carchiolo, A. Faro, O. Mirabella, G. Pappalardo, G. Scollo, "A LOTOS Specification of the PROWAY Highway Service", IEEE Trans. on Computers, Vol. C-35, No. 11, pp. 949-968, November 1986.

[13] W.F. Chan, K. Turner, "The Daemon Game in LOTOS", in: ESTELLE, LOTOS, SDL Draft Examples, Joint Meeting ISO/CCITT (ISO/TC97/SC21/WG1/FDT - CCITT $\mathrm{X} / 3$ ), Turin, December 15-19, 1986.

[14] R. De Nicola, "Extensional Equivalences for Transition Systems", Acta Informatica, Vol. 24, pp. 211-237, 1987.

[15] R. De Nicola, M. Hennessy, "Testing Equivalences for Processes", Theoret. Comput. Sci., Vol. 34, pp. 83-133, North Holland, Amsterdam, 1984.

[16] H. Ehrig, B. Mahr, Fundamentals of Algebraic Specification 1, Springer-Verlag, Berlin, 1985.

[17] ESPRIT/PANGLOSS, Parallel Architectures Networking Gateways Linking OSI Systems. ESPRIT Project 890.

[18] ESPRIT/SEDOS, Software Environment for the Design of Open Distributed Systems. ESPRIT Project ST410.

[19] J.A. Goguen, J.W. Thatcher, E.G. Wagner, "An Initial Algebra Approach to the Specification, Correctness and Implementation of Abstract Data Types", IBM Research Report RC 6487, 1976. Also: Current Trends in Programming Methodology IV: Data Structuring, R. Yeh (Ed), Prentice Hall, 1978.

[20] J. Guttag, "Abstract Data Types and the Development of Data Structures", Communications of the ACM, Vol. 20, N. 6, June 1977.

[21] M. Hennessy, R. Milner, "Algebraic Laws for Nondeterminism and Concurrency", Journal of ACM, Vol. 32, No. 1, pp. 137-161, 1985.

[22] C.A.R. Hoare, Communicating Sequential Processes, Prentice-Hall Intl., 1985.

[23] J. Hopcroft, J. Ullman, Introduction to Automata Theory, Languages, and Computation, Addison-Wesley 1979.

[24] ISO - Information Processing Systems - "Basic Reference Model for Open Systems Interconnection”, IS 7498, 1983.

[25] ISO - Information Processing Systems - Open Systems Interconnection - "Connection Oriented Transport Protocol Specification”, IS 8073, 1986.

[26] ISO - Information Processing Systems - Open Systems Interconnection - "ESTELLE - A Formal Description Technique Based on Extended State Transition Model", DIS 9074, 1987.

[27] ISO - Information Processing Systems - Open Systems Interconnection - "LOTOS - A Formal Description Technique Based on the Temporal Ordering of Observational Behaviour", DIS 8807, 1987.

[28] ISO/TC 97/SC 21 N1534, "Guidelines for the Application of FDT to OSI Protocols and Services", 1986.

[29] ISO/TC 97/SC 21 N1540, "Potential Enhancements to LOTOS", 1986.

[30] K.G. Larsen, "Context Dependent Bisimulations Between Processes", Ph.D. Thesis, University of Edinburgh, Dept. of Computer Science, May 1986.

[31] A. Marshall, "LOTOS Tools Development”, C3 Progress Report, ESPRIT/SEDOS/C3/WP/20/IK, STC Tech. Ltd., Newcastle-under-Lyme, England, january 1987. 
[32] G. Milne, "CIRCAL and the Representation of Communication, Concurrency and Time", ACM Toplas Vol. 7, No. 2, pp. 270-298, 1985.

[33] R. Milner, A Calculus of Communicating Systems, Lecture Notes in Computer Science, Vol. 92, Springer-Verlag, 1980.

[34] R. Milner, "A Complete Inference System for a Class of Regular Behaviours", Journal of Computers and System Sciences, Vol. 28, No. 3, pp. 439-466, 1984.

[35] R. Milner, "Calculi for Synchrony and Asynchrony, Theor. Comp. Science 25, pp. 267-310, 1983.

[36] E. Najm, "A verification oriented specification in Lotos of the Transport Protocol", proceedings of the IFIP Seventh International Symposium on Protocol Specification, Testing, and Verification, H. Rudin and C. West (eds.), North-Holland, 1987.

[37] D. Park, "Concurrency and Automata on Infinite Sequences", Proc. 5th GI Conference, LNCS 104, pp. $167-183,1981$.

[38] G. Plotkin, "A Structural Approach to Operational Semantics", Lecture Notes, Aarhus University, 1981.

[39] Proceedings of the IEEE - Special issue on OSI, Vol. 71. No. 12, Dec. 1983.

[40] J. Quemada, Data Link Service LOTOS Specification, SEDOS/C1/6\& 7/M, December 1986.

[41] G. Scollo, "Formal Description in LOTOS of the OSI
Transport Protocol (Version 9), ESPRIT/SEDOS/C1/ WP/41/T, March 1987.

[42] G. Scollo, F. Minissale, "On the Specification in LOTOS of OSI Protocols", in: G. Bucci, G. Valle (eds.), Computing '85, Proc. 8th ACM European Conf. ICS '85, Florence, Italy, March 1985, pp. 197-206, North-Holland, 1985.

[43] G. Scollo, G. Pappalardo, L. Logrippo, E. Brinksma, "The OSI Transport Service and its Formal Description in LOTOS", in: L. Csaba, K. Tarnay, T. Szentivanyi (eds.), Computer Network Usage: Recent Experiences, pp. 465-488, North-Holland, Amsterdam, 1986.

[44] A.J. Tocher, "OSI Transport Service: A Constraint-Oriented Specification in LOTOS", ESPRIT/SEDOS/C1/ WP $/ 25 /$ IK, ICL, Kidsgrove, August 1986.

[45] K.J. Turner, "OSI connection-oriented network service: a constraint-oriented specification in extended LOTOS (draft 4), SEDOS/C1/WP/15/IK, ICL Kidsgrove, England, May 1986.

[46] P. Van Eijk, "Software Tools for the Specification Language LOTOS", Ph.D. Thesis, University of Twente January 1988.

[47] M. Van Sinderen, "Draft formal specification of the OSI connection-oriented session service in LOTOS (version 5)", SEDOS/C1/WP/35/T, November 1986. 